Pacific Journal of Mathematics

FINITE WEIGHT PROJECTIONS IN YON NEUMANN

Herbert Paul halfekn, Victor Kaftal and László Zsidó 


\title{
FINITE WEIGHT PROJECTIONS IN VON NEUMANN ALGEBRAS
}

\author{
Herbert Halpern, Victor Kaftal and LÁszló Zsidó
}

The ideal of definition of a faithful semifinite normal weight on a countably decomposable von Neumann algebra is the set generated by all positive elements of finite weight. The set is a hereditary left ideal and therefore contains projections. In this paper the family of weights whose ideals of definition form projection lattices is completely characterized. These weights are the ones that are comparable to a combination of traces and normal functionals. A central spectral resolution is introduced and used to analyze the Radon-Nikodym derivatives of a weight with regard to a trace. Also introduced are two parameters that measure whether the ideal of definition contains two projections of least upper bound 1 and how close the weight is to being a trace respectively.

1. Introduction. The set of the projections of a two-sided ideal in a von Neumann algebra is a lattice because the set of projections is hereditary and closed under the Murray-von Neumann equivalence relation (if it contains a projection, it contains also all the projections in the algebra majorized by or equivalent to the projection [16]). The situation is quite different if we take a one-sided ideal. While still hereditary $[10, \S 1.5 .2]$, a one-sided ideal that is closed under the equivalence relation for projections is a two-sided ideal. However, there are interesting cases of one-sided ideals where the set of projections is nevertheless a lattice, e.g. the right ideal of "finite rank" operators in a type $\mathrm{III}_{\lambda}$ factor (cf. [5, §3]).

There are one-sided ideals whose set of projections is not a lattice. For example, if $\varphi$ is a faithful semifinite normal weight (henceforth f.s.n. for short) on a von Neumann algebra $R$, then

$$
N_{\varphi}=\left\{x \in R \mid \varphi\left(x^{*} x\right)<\infty\right\}
$$

is a left ideal and

$$
M_{\varphi}=\operatorname{span}\left\{x \in R^{+} \mid \varphi(x)<\infty\right\}=N_{\varphi}^{*} N_{\varphi}
$$

is a hereditary algebra $[15,10.14]$. In $[5, \S 7]$ the first two named authors applied a result by $U$. Haagerup ([2], cf. [13, 30.12]) relating to infinite weights in type $\mathrm{III}_{\lambda}$ factors to construct two projections 
$p, q$ in $M_{\varphi}$ whose least upper bound $p \vee q$ was not in $M_{\varphi}$. In [6, Example 5.4], the same authors used a different technique employing the discrete crossed product decomposition of a type $\mathrm{III}_{\lambda}$ factor $R$ to construct two projections $p, q$ in $M_{\varphi}$ with $p \vee q=1$. Both constructions made use of the fact that the least upper bound of two distinct rank-one projections in $M_{2}(\mathbb{C})$ (the $2 \times 2$ complex matrix algebra) is the identity. A similar result, also depending on a $2 \times 2$ matrix construction, was obtained by A. Amann and the third named author in [1] for the null ideal

$$
L_{\omega}=\left\{x \in R \mid \omega\left(x^{*} x\right)=0\right\}
$$

of a singular state $\omega \in R^{*}$ from quantum mechanics.

Motivated by the analogy between the ideals $L_{\omega}$ for a singular state $\omega$ and $N_{\varphi}$ for an infinite weight $\varphi$, we determine in this paper necessary and sufficient conditions under which the set $P\left(M_{\varphi}\right)$ of the projections of the ideal of definition $M_{\varphi}$ of an f.s.n. weight $\varphi$ on a von Neumann algebra $R$ is a lattice. Without loss of generality we may always assume that the weights that we consider are faithful (otherwise we could pass to the algebra reduced to the support of the weight). Since all the projections in $M_{\varphi}$ are $\sigma$-finite and since the least upper bound of two $\sigma$-finite projections is also $\sigma$-finite, we reduce our considerations to $\sigma$-finite von Neumann algebras.

Our main result is:

TheOREM 1. Let $R$ be a $\sigma$-finite von Neumann algebra and let $\varphi$ be a f.s.n. weight on $R$. Then $P\left(M_{\varphi}\right)$ is a lattice if and only if there is a decomposition of the identity into mutually orthogonal central projections $e+f+g=1$ such that $R_{f}$ is a semifinite algebra and $R_{g}$ is a direct sum of type $\mathbf{I}_{\infty}$ factors equipped with the f.s.n. trace $\operatorname{Tr}$ (the direct sum of the canonical traces on the factors) so that

(a) $\varphi$ restricted to $R_{e}$ is a finite functional,

(b) $P\left(M_{\varphi(f .)}\right)=P\left(M_{\tau}\right)$ for some f.s.n trace $\tau$ on $R_{f}$, and

(c) $P\left(M_{\varphi(g \cdot)}\right) \subset P\left(M_{\mathrm{Tr}}\right)$.

We define two parameters

$$
I(\varphi)=\inf \{\varphi(p+q) \mid p \vee q=1, p, q \text { projections in } R\}
$$

and

$$
J(\varphi)=\sup \left\{\frac{\varphi(p \vee q)}{\varphi(p+q)} \mid 0 \neq p, q \in P\left(M_{\varphi}\right)\right\}
$$

to study the lattice properties of $P\left(M_{\varphi}\right)$. The first $I(\varphi)$ measures how close the identity 1 is to being the least upper bound of projections 
in $P\left(M_{\varphi}\right)$ while the second $J(\varphi)$ measures how close $\varphi$ is to being a trace.

For a type III algebra we see from Theorem 1 that $P\left(M_{\varphi}\right)$ is a lattice if and only if $\varphi$ is a finite functional. For semifinite algebras we see from Theorem 1 that the situation is more complex. Here we exploit the properties of the Radon-Nikodym derivative $h$ of $\varphi$ with respect to a f.s.n. trace $\tau$ (cf. [11]). The Radon-Nikodym derivative $h$ is a positive selfadjoint (possibly unbounded) operator affiliated with the centralizer algebra

$$
R^{\varphi}=\left\{x \in R \mid \sigma_{t}^{\varphi}(x) \equiv x\right\}
$$

such that $\varphi(x)=\tau(h x)$ for all $x \in R^{+}$, where

$$
\tau(h x)=\tau\left(x^{1 / 2} h x^{1 / 2}\right)=\lim _{n} \tau\left(x^{1 / 2} h \chi(-\infty, n)(h) x^{1 / 2}\right) .
$$

Here $\chi E(h)$ denotes the spectral projection of $h$ corresponding to the Borel set $E$. (When there is no possibility of confusion, we shall drop the reference to $h$ and just write $\chi E$. We generally use the whole real line as the domain of the spectral resolution even for positive operators since it allows us to unify the notation when we analyze the essential central spectrum of an unbounded operator. The essential central spectrum $Z-\sigma^{e}(x)$ for a bounded operator $x$ has been developed in [4] and [14]. Here we extend the concept of essential central spectrum to an unbounded selfadjoint operator $h$ via the spectral resolution and arrive at a concept of central intervals. We calculate the parameters $I(\varphi)$ and $J(\varphi)$ and show the former is related to the central essential spectrum of the Radon-Nikodym derivative while the latter is related to the spread in the essential spectrum of the RadonNikodym derivative. In particular, we have that $J(\varphi)=1$ if and only if $\varphi$ is a trace.

We analyze $P\left(M_{\varphi}\right)$ for finite algebras separately. We find a canonical trace $\tau_{\varphi}$ associated to a f.s.n. weight $\varphi$ and show that $P\left(M_{\varphi}\right)$ is a lattice if and only if $P\left(M_{\varphi}\right)$ and $P\left(M_{\tau_{\varphi}}\right)$ coincide.

One of the tools used throughout this paper is the notion of $\varphi$ semifinite projection, i.e., a projection $p$ such that the restriction of $\varphi$ to $R_{p}$ is still semifinite. We also use a $2 \times 2$ matrix construction to obtain the sum of two orthogonal projections as a least upper bound of two projections only one of which has to be controlled.

A few remarks about our notations: the algebra $R$ operates on the Hilbert space $H$ and has identity $1 ; Z$ denotes the center of $R, \widehat{Z}^{+}$denotes the extended positive part of $Z$, and $\widetilde{Z}$ denotes the 
selfadjoint operators on $H$ affiliated with $Z$. Recall that under the identification of $Z$ with $L^{\infty}\{\Gamma, \mu\}$, where $\Gamma$ is a locally compact space and $\mu$ is a positive Radon measure, the set $\widehat{Z}^{+}$coincides with the set of $\mu$-measurable extended real valued nonnegative functions and it is closed under least upper bounds. On the other hand, the set $\widetilde{Z}$ coincides with the set of all real valued measurable functions that are finite almost everywhere. For every projection $p$ in $R, R_{p}$ is the algebra $p R p$ restricted to the space $p H$ and $c(p)$ is the central support of $p ; p \vee q$ and $p \wedge q$ are the least upper bound and the greatest lower bound respectively of the projections $p$ and $q ; R(x)$ and $N(x)$ are respectively the left support (i.e. the range projection) and the null projection of an operator $x ; k^{+}$and $k^{-}$are the positive part and the negative part respectively of a selfadjoint (possibly unbounded) operator $k$. For the rest of our notations we refer the reader to [15].

2. $\varphi$-semifinite projections. Let $R$ be a $\sigma$-finite (i.e. countably decomposable) von Neumann algebra and let $\varphi$ be a faithful semifinite normal weight on $R$ (f.s.n for short). Let

$$
M_{\varphi}=\operatorname{span}\left\{x \in R^{+} \mid \varphi(x)<\infty\right\} .
$$

Definition 2.1. A projection $p \in R$ is said to be $\varphi$-semifinite ( $\varphi$-s. for short) if the restriction of $\varphi$ to $R_{p}$ is semifinite. The projection $p$ is said to be $\varphi$-purely infinite if the restriction of $\varphi$ to $R_{p}$ assumes only the values $\{0, \infty\}$.

Notice that the restriction of $\varphi$ to $R_{p}$ is always a faithful and normal weight, and it is semifinite if and only if $M_{\varphi} \cap R_{p}$ is $\sigma$-weakly dense in $R_{p}$.

We shall often use the following criterions for a projection to be $\varphi$-S.

LEMMA 2.2. Let $p \in R$ be a projection; then the following conditions are equivalent.

(i) $p$ is $\varphi$-s.

(ii) There is an $x \in M_{\varphi}^{+}$such that $R(x)=p$.

(iii) There is a sequence of mutually orthogonal projections $p_{n}$ in $M_{\varphi}$ such that $p=\sum p_{n}$.

Proof. Let $p$ be $\varphi$-s. We can find a countable strongly dense subset $\left\{x_{n}\right\}$ in the unit ball of $M_{\varphi} \cap R_{p}^{+}$because the unit ball of $R_{p}^{+}$is metrizable in the strong operator topology $[8,5.7 .46]$. Then the series 
$\sum 2^{-n}\left(1+\varphi\left(x_{n}\right)\right)^{-1} x_{n}$ converges uniformly to an operator $x \in R_{p}^{+}$. We see that $R(x)=p$ due to the density of the set $\left\{x_{n}\right\}$. By the normality of $\varphi$, we get that

$$
\varphi(x)=\sum 2^{-n}\left(1+\varphi\left(x_{n}\right)\right)^{-1} \varphi\left(x_{n}\right)<\infty .
$$

Assume now that there is an $x \in M_{\varphi}^{+}$such that $R(x)=p$. The spectral projections $p_{n}=\chi\left[n^{-1},(n+1)^{-1}\right)(x)$ of $x$ corresponding to the intervals $\left[n^{-1},(n+1)^{-1}\right)$ are mutually orthogonal with sum equal to $R(x)$. Moreover, each projection $p_{n}$ is in $M_{\varphi}$ since

$$
\varphi\left(p_{n}\right) \leq n \varphi\left(p_{n} x\right) \leq n \varphi(x)<\infty .
$$

Thus, the projection $p$ is the sum of the sequence of mutually orthogonal projections $\left\{p_{n}\right\}$ in $M_{\varphi}$.

Finally, if $p$ is the sum of a sequence of mutually orthogonal projections $\left\{p_{n}\right\}$ in $M_{\varphi}$, let $q_{n}=p_{1}+\cdots+p_{n}$. The subset $U\left(R_{q_{n}}\right)$ of $M_{\varphi}$ is $\sigma$-weakly dense in $R_{p}$.

Given a projection $p$ in $R$, we can find a maximal sequence of mutually orthogonal $\varphi$-s. subprojections $\left\{p_{n}\right\}$ of $p$. By maximality, the projection $p-\sum p_{n}$ is $\varphi$-purely infinite. So $p$ can be decomposed into the sum of a $\varphi$-s. and a $\varphi$-purely infinite projection. This decomposition is in general not unique. Indeed, the identity operator is $\varphi$-s. by definition but may be decomposed as a nontrivial sum of a $\varphi$-s. and a $\varphi$-purely infinite projection (see remarks after Proposition 2.4).

In finite algebras there are no $\varphi$-purely infinite projections.

Proposition 2.3. Every projection in a finite von Neumann algebra is $\varphi$-semifinite.

Proof. Let $p \neq 1$ be an arbitrary nonzero projection in the finite von Neumann algebra $R$. Let $\tau$ be a f.n. finite trace with $\tau(1)=1$. Let $\varphi$ be a f.s.n. weight on $R$ and let $h$ be the Radon-Nikodym derivative of $\varphi$ with respect to $\tau$. By the normality of the trace there is some $n>0$ and a spectral projection $q=\chi[0, n)(h)$ such that $\tau(q)>1-\tau(p)$. By the Parallelogram Law we have that

$$
p \vee q-p \sim q-p \wedge q \text {. }
$$

Then we have that

$$
1-\tau(p) \geq \tau(p \vee q)-\tau(p)=\tau(q)-\tau(p \wedge q)>1-\tau(p)-\tau(p \wedge q) .
$$


This shows that the projection $p \wedge q$ is not 0 . Moreover

$$
\varphi(p \wedge q) \leq \varphi(q)=\tau(h q) \leq n \tau(q)<\infty .
$$

Thus, we have shown that every nonzero projection in $R$ majorizes a nonzero projection in $M_{\varphi}$. By a maximality argument, we have that every projection can be written as the sum of a sequence of mutually orthogonal projections in $M_{\varphi}$. Thus, every projection is $\varphi$-s. by Lemma 2.2.

If $\varphi$ is a trace, then clearly there are also no $\varphi$-purely infinite projections. In the case of $B(H)$, there are $\varphi$-purely infinite projections if and only if the Radon-Nikodym derivative $h$ of $\varphi$ with respect to the canonical trace $\operatorname{tr}$ is unbounded. Indeed, on the one hand, if $h$ is bounded then any finite projection is in $M_{\varphi}$. On the other hand, if $h$ is unbounded, then there is a unit vector $\xi$ not in the domain of $h^{1 / 2}$. Setting $p$ equal to the one dimensional projection with range span $\xi$, and $h_{n}=h \chi[0, n)(h)$ we get that

$$
\begin{aligned}
\operatorname{tr}(h p) & =\lim _{n} \operatorname{tr}\left(p h_{n} p\right) \\
& =\lim _{n}\left(h_{n} \xi, \xi\right) \\
& =\lim _{n}\left\|h_{n}^{1 / 2} \xi\right\|^{2}=\infty .
\end{aligned}
$$

More generally, let $\varphi=\psi \otimes \operatorname{tr}(h \cdot)$ be a f.s.n weight on a von Neumann algebra of the form $R \otimes B(H)$. Again if $h$ is unbounded, then we have

$$
\varphi(q \otimes p)=\psi(q) \operatorname{tr}(h p)=\infty
$$

for any nonzero projection $q \in R$. From this we conclude that $1 \otimes p$ is $\varphi$-purely infinite.

The $\varphi$-semifinite projections exhibit some of the standard properties associated with semifinite projections.

Proposition 2.4. Let $\varphi$ be f.s.n. weight on the von Neumann algebra $R$. Then

(i) The supremum of a countable set of $\varphi$-s. projections is $\varphi$-s.

(ii) Let $p$ be a $\varphi$-s. projection and let $a \in R^{\varphi}$; then $R\left(a p a^{*}\right)$ is $\varphi-s$.

(iii) Let $p, q$ be two $\varphi$-s. projections with $p \geq q$. If $q \in M_{\varphi}$, then $p-q$ is $\varphi$-s. 
(iv) Let $R$ be semifinite, let $\tau$ be a f.s.n. trace on $R$ and let $h$ be the Radon-Nikodym derivative of $\varphi$ with respect to $\tau$; then a projection $p$ is $\varphi$-s. if and only if php is selfadjoint.

Proof (i). Let $\left\{p_{n}\right\}$ be a sequence of $\varphi$-s. projections. Let $\left\{p_{n m}\right\}$ be sequences of projections in $M_{\varphi}$ with $p_{n}=\sum_{m} p_{n m}$. Then

$$
x=\sum_{m, n} 2^{-m-n}\left(1+\varphi\left(p_{n m}\right)\right)^{-1} p_{n m}
$$

is an element in $M_{\varphi}$ and has range projection equal to $\sup p_{n}$. By Lemma 2.2, this implies that $\sup p_{n}$ is $\varphi$-s.

Proof (ii). By Lemma 2.2 we can find an $x \in M_{\varphi}^{+}$with $R(x)=p$. Since $a x a^{*} \in M_{\varphi}$ because $M_{\varphi}$ is an $R^{\varphi}$-module, and since $R\left(a p a^{*}\right)=$ $R\left(a x a^{*}\right)$, we see that $R\left(a p a^{*}\right)$ is $\varphi$-s.

Proof (iii). By Lemma 2.2 we can find an $x \in M_{\varphi}^{+}$with $R(x)=p$. Then

$$
(p-q) x(p-q) \leq 2 p x p+2 q x q \leq 2 x+2\|x\| q,
$$

whence $(p-q) x(p-q) \in M_{\varphi}^{+}$. Since $p-q=R((p-q) x(p-q))$, we conclude, again by Lemma 2.2 , that $p-q$ is $\varphi$-s.

Proof (iv). Assume first that $p \in M_{\varphi}$ and let $h_{n}=h \chi[0, n)(h)$. The sequence $p h_{n} p$ increases monotonically and hence it has a limit $k$ belonging to the extended positive part $\widehat{M}^{+}$of $M$, and $\hat{k}$ has a unique representation $\hat{k}=k+\infty q$ where $k=q^{\perp} k q^{\perp}$ is a positive selfadjoint operator affiliated with $M$ and $q \in M$ is a projection [2, Remarks after Definition 1.3, Lemma 1.4 and Theorem 1.5]. Then $\tau$ has an extension $\hat{\tau}$ to $\widehat{M}^{+}$and

$$
\varphi(p)=\lim _{n} \tau\left(p h_{n} p\right)=\hat{\tau}(\hat{k})=\tau(k)+\infty \tau(q),
$$

whence $q=0$ [2, Proposition 1.10]. Thus $p h_{n} p \uparrow k$ in the sense that

$$
\left(p h_{n} p \xi, \xi\right) \uparrow \begin{cases}\left\|k^{1 / 2} \xi\right\|^{2} & \text { if } \xi \in D\left(k^{1 / 2}\right), \\ \infty & \text { if } \xi \notin D\left(k^{1 / 2}\right) .\end{cases}
$$

Since we also have

$$
\left(p h_{n} p \xi, \xi\right) \uparrow \begin{cases}\left\|h^{1 / 2} p \xi\right\|^{2} & \text { if } \xi \in D\left(h^{1 / 2} p\right), \\ \infty & \text { if } \xi \notin D\left(h^{1 / 2} p\right),\end{cases}
$$


we obtain that $D\left(k^{1 / 2}\right)=D\left(h^{1 / 2} p\right)$ and hence that $h^{1 / 2} p$ is densely defined. (We can actually show that $k=p h p$, but we don't need this fact here.)

Assume now that $p$ is $\varphi$-s., so that by Lemma 2.2 there is a sequence of mutually orthogonal projections $p_{k} \in M_{\varphi}$ such that $p=\sum p_{k}$. We have just proven that $h^{1 / 2} p_{k}$ is densely defined for each $k$, and a routine argument shows that then $h^{1 / 2} p$ too is densely defined. Clearly, $h^{1 / 2} p$ is closed and since $\left(h^{1 / 2} p\right)^{*} \supseteq p h^{1 / 2}$, we see that $p h^{1 / 2}$ too is closed. It is easy to verify that $\left(p h^{1 / 2}\right)^{*}=h^{1 / 2} p$, and since $p h^{1 / 2}=\left(p h^{1 / 2}\right)^{* *}$, we have also $\left(h^{1 / 2} p\right)^{*}=p h^{1 / 2}$. Therefore, by [8, Theorem 2.7.8 (v)], we obtain that $\left(h^{1 / 2} p\right)^{*} p h^{1 / 2}=p h p$ is selfadjoint.

Conversely, if $p h p$ is selfadjoint then $\tau(p h p)$ is an s.n. weight on $R$ which coincides with $\varphi$ on $R_{p}$.

In general the condition $q \in M_{\varphi}$ in (iii) cannot be relaxed. We can show this by refining the example after Proposition 2.3. Let $R=$ $B(H)$, let $h$ be a positive injective selfadjoint unbounded operator on $H$, and let $\varphi$ be the f.s.n. weight defined by $\varphi=\operatorname{tr}(h \cdot)$. Working with the spectral resolution of $h$, we can find an orthonormal basis $\left\{\xi_{n}\right\}$ for $H$ such that $\xi_{1} \notin D\left(h^{1 / 2}\right)$ while $\left\{\xi_{n} \mid n \geq 2\right\}$ is contained in $D\left(h^{1 / 2}\right)$. Setting $p_{n}$ equal to the one dimensional projection of $H$ on the subspace generated by $\xi_{n}$, we get that the $\varphi$-purely infinite projection $p_{1}$ can be written as $p_{1}=1-\sum\left\{p_{n} \mid n \geq 2\right\}$ whereas 1 and $\sum\left\{p_{n} \mid n \geq 2\right\}$ are $\varphi$-s.

Notice also that by (ii) every projection in $R^{\varphi}$ and in particular every central projection is $\varphi$-s.

LEMMA 2.5. Let $\varphi$ be a f.s.n. weight on $R$; then, for every projection $p$ in $R$ and every $\varphi$-s. projection $q$ with $p \prec q$, there is $a \varphi$-s. projection $q^{\prime}$ with $q^{\prime} \leq q$ and $p \sim q^{\prime}$.

Proof. There is a projection $p^{\prime} \sim p$ with $p^{\prime} \leq q$. So there is no loss of generality in the assumption that $p \leq q$. Since the weight $\varphi$ restricted to the algebra $R_{q}$ is f.s.n., we may assume also that $q=1$. If $p$ were properly infinite, then it would be equivalent to its central support $c(p)$, which is $\varphi$-s. by Proposition 2.4(i), and if $R$ were finite, then $p$ would be $\varphi$-s. by Proposition 2.3. Thus we can assume that $p$ is a finite projection of central support 1 and that $R$ is a properly infinite semifinite algebra. Let $\Phi$ be a faithful normal operator valued trace on $R$ with $\Phi(p)=1$, let $\omega$ be a f.n. state on $Z$, let $\tau=\omega \circ \Phi$ 
be the corresponding f.s.n. (scalar) trace, and let $h$ be the RadonNikodym derivative of $\varphi$ with respect to $\tau$. We may find a sequence $\left\{n_{i}\right\}$ of integers and a sequence $\left\{e_{i}\right\}$ of orthogonal central projections of sum 1 with $e_{i} \leq \Phi\left(\chi\left(-\infty, n_{i}\right)(h)\right) e_{i}$. Then we have that

$$
\Phi(p)=1 \leq \sum \Phi\left(\chi\left(-\infty, n_{i}\right)(h)\right) e_{i}=\Phi\left(\sum \chi\left(-\infty, n_{i}\right)(h) e_{i}\right) .
$$

This proves that

$$
p \sim q^{\prime} \leq \sum \chi\left(-\infty, n_{i}\right)(h) e_{i}
$$

Since

$$
\varphi\left(q^{\prime} e_{i}\right)=\tau\left(h q^{\prime} e_{i}\right) \leq n_{i} \tau\left(q^{\prime} e_{i}\right) \leq n_{i} \tau(p)<\infty,
$$

we see that $q^{\prime}=\sum q^{\prime} e_{i}$ is $\varphi$-s. by Lemma 2.2 .

3. A $2 \times 2$ matrix construction. Now we can start to investigate the condition on a f.s.n. weight $\varphi$ under which the set $P\left(M_{\varphi}\right)$ of projections of $M_{\varphi}$ is a lattice.

LEMMA 3.1. Let $p, s$ be two equivalent and orthogonal $\varphi$-s. projections in $R$ with $p \in M_{\varphi}$. Then for every $\varepsilon>0$ there is a projection $q \in M_{\varphi}$ such that $p \vee q=p+s$ and $\varphi(q)<\varphi(p)+\varepsilon$.

Proof. We actually obtain a projection $q$ with $q \sim p \sim s$. By Lemma 2.2 we can decompose $s$ into a sum $\sum s_{n}$ of mutually orthogonal projections $s_{n}$ in $M_{\varphi}$. This decomposition induces a corresponding partition of $p$ into the sum of mutually orthogonal projections $p=\sum p_{n}$ with $p_{n} \sim s_{n}$. There are partial isometries $u_{n} \in R$ implementing this equivalence, i.e.,

$$
u_{n}^{*} u_{n}=p_{n} \text { and } u_{n} u_{n}^{*}=s_{n} .
$$

Since $p_{n}$ and $s_{n}$ are in $M_{\varphi}$, so are also $u_{n}$ and $u_{n}^{*}$, and by the Cauchy-Schwarz inequality $\left|\varphi\left(u_{n}\right)\right|$ and $\left|\varphi\left(u_{n}^{*}\right)\right|$ are both bounded by $\sqrt{\varphi\left(p_{n}\right) \varphi\left(s_{n}\right)}$. Choose also a sequence $\delta_{n} \in(0,1)$ such that

$$
\sum\left\{\delta_{n} \varphi\left(s_{n}\right)+2 \sqrt{\delta_{n} \varphi\left(p_{n}\right) \varphi\left(s_{n}\right)}\right\}<\varepsilon .
$$

Let $R_{n}=\operatorname{span}\left\{p_{n}, s_{n}, u_{n}, u_{n}^{*}\right\}$; then $R_{n}$ is a subalgebra of $R$ naturally isomorphic to $M_{2}(\mathbb{C})$. Let $q_{n} \in R_{n}$ be the projection corresponding to

$$
\left[\begin{array}{cc}
1-\delta_{n} & \sqrt{\delta_{n}\left(1-\delta_{n}\right)} \\
\sqrt{\delta_{n}\left(1-\delta_{n}\right)} & \delta_{n}
\end{array}\right]
$$


i.e.,

$$
q_{n}=\left(1-\delta_{n}\right) p_{n}+\delta_{n} s_{n}+\sqrt{\delta_{n}\left(1-\delta_{n}\right)}\left(u_{n}+u_{n}^{*}\right) .
$$

Then $p_{n} \neq q_{n}$ because $\delta_{n} \neq 0$, and hence $p_{n} \vee q_{n}=p_{n}+s_{n}$. Let $q=\sum q_{n}$; then it is easy to verify that

$$
p \vee q=\sum\left(p_{n} \vee q_{n}\right)=\sum\left(p_{n}+s_{n}\right)=p+s .
$$

On the other hand,

$$
\begin{aligned}
\varphi\left(q_{n}\right) & \leq\left(1-\delta_{n}\right) \varphi\left(p_{n}\right)+\delta_{n} \varphi\left(s_{n}\right)+\sqrt{\delta_{n}\left(1-\delta_{n}\right)}\left(\left|\varphi\left(u_{n}\right)\right|+\left|\varphi\left(u_{n}^{*}\right)\right|\right) \\
& \leq \varphi\left(p_{n}\right)+\delta_{n} \varphi\left(s_{n}\right)+2 \sqrt{\delta_{n} \varphi\left(p_{n}\right) \varphi\left(s_{n}\right)}
\end{aligned}
$$

so that

$$
\varphi(q) \leq \sum \varphi\left(p_{n}\right)+\sum\left\{\delta_{n} \varphi\left(s_{n}\right)+2 \sqrt{\delta_{n} \varphi\left(p_{n}\right) \varphi\left(s_{n}\right)}\right\} \leq \varphi(p)+\varepsilon .
$$

Since $\varphi(p)<\infty$ and $q$ is $\varphi$-s. imply that $p \vee q-p$ is $\varphi$-s. due to Proposition 2.4(i), the hypothesis that $s$ is $\varphi$-s. cannot be avoided in Lemma 3.1.

Proposition 3.2. The set of projections $P\left(M_{\varphi}\right)$ is not a lattice if and only if there are two equivalent and orthogonal $\varphi$-s. projections $r$ and $s$ in $R$ such that $r \in M_{\varphi}$ and $s \notin M_{\varphi}$.

Proof. First suppose that $P\left(M_{\varphi}\right)$ is not a lattice. Let $p$ and $q$ be two projections in $M_{\varphi}$ such that $p \vee q$ is not in $M_{\varphi}$. Since

$$
(p-p \wedge q) \vee q=p \vee q \text { and }(p-p \wedge q) \wedge q=0 \text {, }
$$

by passing to subprojections if necessary, we may assume that $p \wedge q=$ 0 . By the Comparison Theorem there is a central projection $e$ such that

$$
p e \sim q_{1} \leq q e
$$

and

$$
q(1-e) \sim p_{1} \leq p(1-e) .
$$

The projections $q e$ and $p(1-e)$ are in $M_{\varphi}$ and so the projection $r=p_{1}+q_{1}$ is in $M_{\varphi}$ too. However, the projection

$$
s=(p \vee q-q) e+(p \vee q-p)(1-e)
$$

is $\varphi$-s. (Proposition 2.4(i) and (iii)) but it is not in $M_{\varphi}$. Also, $s$ is orthogonal to $r$ and it is equivalent to it via the Parallelogram Law

$$
(p \vee q-q) e+(p \vee q-p)(1-e) \sim p e+q(1-e) \sim p_{1}+q_{1}
$$

due to the assumption that $p \wedge q=0$. 
Now suppose that $r$ and $s$ are orthogonal equivalent $\varphi$-s. projections with $r \in M_{\varphi}$ and $s \notin M_{\varphi}$. By Lemma 3.1 there is a projection $q$ in $R$ with $r \vee q=r+s$ and with $\varphi(q) \leq \varphi(r)+1<\infty$. Since $r+s \notin M_{\varphi}$, we see that $P\left(M_{\varphi}\right)$ is not a lattice.

Proposition 3.3. Let $R$ be a type III algebra and let $\varphi$ be a f.s.n. weight on $R$. Then the set $P\left(M_{\varphi}\right)$ is a lattice if and only if $\varphi$ is finite.

Proof. The condition is clearly sufficient. Assume now that $P\left(M_{\varphi}\right)$ is a lattice. Let $\left\{p_{n}\right\}$ be a maximal set (necessarily countable since $R$ is $\sigma$-finite) of nonzero projections in $P\left(M_{\varphi}\right)$ with mutually orthogonal central supports. Since $e=1-c\left(\sum p_{n}\right)$ is $\varphi$-s. by Proposition 2.4(ii), by the maximality of the family we see that $e=0$. Since we may write each $p_{n}$ as the sum of a sequence of mutually orthogonal equivalent projections, we may assume without loss of generality that $\varphi\left(p_{n}\right)<$ $2^{-n}$ for every $n=1,2, \ldots$. The projection $p=\sum p_{n}$ is then a projection in $M_{\varphi}$ of central support 1 . Since $R$ is type III and $\sigma$ finite, by passing to a subprojection of $p$, we may assume that $p \sim$ $1-p \sim 1$. Since $1-p$ is $\varphi$-s. due to Proposition 2.4 , by Lemma 3.1 we can find a projection $q$ such that

$$
p \vee q=p+(1-p)=1 \text { and } \varphi(q) \leq \varphi(p)+1<\infty
$$

By assumption the set $P\left(M_{\varphi}\right)$ is a lattice, and therefore, $\varphi(1)<\infty$. Thus, the weight $\varphi$ is a finite normal functional.

We now introduce two numbers associated with every f.s.n weight.

Definition 3.4. Let $\varphi$ be a f.s.n. weight on the von Neumann algebra $R$. Then let

$$
I(\varphi)=\inf \{\varphi(p+q) \mid p \vee q=1, p, q \text { projections in } R\},
$$

and

$$
J(\varphi)=\sup \left\{\frac{\varphi(p \vee q)}{\varphi(p+q)} \mid p, q \in M_{\varphi} \text { and } p+q \neq 0\right\} .
$$

We have already seen that

$$
I(\varphi)=\inf \{\varphi(p+q) \mid p \vee q=1, p \wedge q=0, p, q \text { projections in } R\}
$$

since $(p-p \wedge q) \vee q=p \vee q$ and $(p-p \wedge q) \wedge q=0$.

We use the numbers $I(\varphi)$ and $J(\varphi)$ to determine when $P\left(M_{\varphi}\right)$ is a lattice. 
Proposition 3.5. If $R$ is a type III algebra then $I(\varphi)=0$ and $J(\varphi)=\infty$.

Proof. By slightly changing the proof of Proposition 3.3, given $\varepsilon>$ 0 , we can find two projections $p$ and $q$ in $R$ with $p \vee q=1$ and $\varphi(p)+\varphi(q)<\varepsilon$. This proves that $I(\varphi)=0$.

We can now see that $J(\varphi)=\infty$ since the supremum of $\varphi(p \vee q) / \varphi(p+q)$ over the set of projections $p$ and $q$ with $p$ and $q$ in $M_{\varphi}$ and $p \vee q=1$ is already $\infty$.

Actually, we can see that $J(\varphi)=\infty$ as soon as $R$ has a nonzero type III direct summand. Also $I(\varphi)=0$ if $\varphi$ is finite and $R$ is properly infinite.

4. The semifinite case. Comparison of $M_{\varphi}$ and $M_{\tau}$. We begin with a discussion of "central intervals" which we need throughout the rest of the present work. Let $Z$ be the center of the von Neumann algebra $R$ and $\widetilde{Z}$ be the set of all densely defined selfadjoint elements affiliated with $Z$. For each $z \in \widetilde{Z}$, there is a sequence $\left\{e_{n}\right\}$ of mutually orthogonal projections in $Z$ of sum 1 such that $z e_{n}$ is in $Z$ for every $n$. If $x$ and $y$ are in $\widetilde{Z}$, then we write $x<y$ (respectively, $x \leq y$ ) if there is a sequence $\left\{e_{n}\right\}$ of mutually orthogonal projections in $Z$ of sum 1 such that $x e_{n}$ and $y e_{n}$ are bounded and $x e_{n}<y e_{n}$ (respectively, $x e_{n} \leq y e_{n}$ ) for every $n$.

Now let $h$ be a selfadjoint element affiliated with $R$ and let $\chi$ be the spectral resolution of $h$. Let $\left\{e_{n}\right\}$ and $\left\{f_{n}\right\}$ be sequences of mutually orthogonal projections in $Z$ of sum 1 and let $\left\{\alpha_{n}\right\}$ and $\left\{\beta_{n}\right\}$ be sequences in $R$. If $\sum \alpha_{n} e_{n} \leq \sum \beta_{n} f_{n}$, we have that

$$
\sum \chi\left(-\infty, \alpha_{n}\right) e_{n} \leq \sum \chi\left(-\infty, \beta_{n}\right) f_{n}
$$

Now let $z \in \widetilde{Z}$. Then the family of all sums of the form $\sum \alpha_{n} e_{n}$ with $\sum \alpha_{n} e_{n}<z$ is upward directed. Thus, the following definition is possible.

Definition 4.1. Let $h$ be a selfadjoint element affiliated with $R$ with spectral resolution $\chi$ and let $z \in \widetilde{Z}$. Then let $\chi(-\infty, z)(h)=$ $\chi(-\infty, z)$ be the least upper bound of the increasing family of projections of the form $\sum \chi\left(-\infty, \alpha_{n}\right) e_{n}$ for all sequences $\left\{e_{n}\right\}$ of central projections of sum 1 and sequences $\left\{\alpha_{n}\right\}$ of real numbers with $\sum \alpha_{n} e_{n}<z$.

We similarly define the other central spectral projection $\chi(z, \infty)$ as the least upper bound of the increasing family of projections of 
the form $\sum \chi\left(\alpha_{n}, \infty\right) e_{n}$ for all sequences $\left\{e_{n}\right\}$ of central projections of sum 1 and sequences $\left\{\alpha_{n}\right\}$ of real numbers with $\sum \alpha_{n} e_{n}>z$. We note that both $\chi(-\infty, z)$ and $\chi(z, \infty)$ are in the von Neumann algebra generated by $Z$ and $h$.

Now let $z \in \widetilde{Z}$ and let $h$ be a selfadjoint element affiliated with $R$. Then the operator $z-h$ is a densely defined operator. In fact, there is a sequence of mutually orthogonal projections $\left\{p_{n}\right\}$ in $R$ of sum 1 commuting with $z$ and $h$ such that $p_{n} H$ is contained in the domain $D(z) \cap D(h)$ of $z-h$. The closure of this operator is a selfadjoint operator affiliated with $R$. We again denote this selfadjoint operator by $z-h$. The operator $z-h$ is affiliated with the von Neumann algebra generated by $Z$ and $h$ as are the positive selfadjoint operators

$$
(z-h)^{+}=(z-h) \chi(0, \infty)(z-h)
$$

and

$$
(z-h)^{-}=-(z-h) \chi(-\infty, 0)(z-h)
$$

(cf. $[8,2.7 .10])$.

We have a different characterization of the central spectral projections.

Proposition 4.2. Let $R$ be a von Neumann algebra and let $h$ be a selfadjoint element affiliated with $R$. Then, for every $z \in \widetilde{Z}$, $\chi(-\infty, z)(h)=R\left((z-h)^{+}\right)$and $\chi(z, \infty)(h)=R\left((z-h)^{-}\right)$.

Proof. We verify only the first relationship. Since $z-h$ is a selfadjoint operator affiliated with the von Neumann algebra generated by $Z$ and $h$, it has a spectral resolution $\chi^{\prime}$ and $R\left((z-h)^{+}\right)=\chi^{\prime}(0, \infty)$. In particular, the spectral resolutions $\chi$ and $\chi^{\prime}$ commute. Now let $\left\{e_{n}\right\}$ be a sequence of central projections of sum 1 and let $\left\{\alpha_{n}\right\}$ be a sequence of real numbers such that $\sum \alpha_{n} e_{n}<z$. There is no loss of generality in the assumption that $z e_{n}-\alpha_{n} e_{n}$ is a bounded invertible positive operator since we may decompose the $e_{n}$ further. Then we have, for $-m<\alpha_{n}$, that

$$
z \chi\left(-m, \alpha_{n}\right) e_{n}-\chi\left(-m, \alpha_{n}\right) h e_{n} \geq z \chi\left(-m, \alpha_{n}\right) e_{n}-\alpha_{n} \chi\left(-m, \alpha_{n}\right) e_{n}
$$

shows that $z \chi\left(-m, \alpha_{n}\right) e_{n}-\chi\left(-m, \alpha_{n}\right) h e_{n}$ is an invertible positive operator on $\chi\left(-m, \alpha_{n}\right) e_{n} H$. Since $\chi\left(-m, \alpha_{n}\right) e_{n}$ commutes with $z-h$, we have that

$$
R\left((z-h)^{+}\right) \geq \chi\left(-m, \alpha_{n}\right) e_{n}
$$


By taking the least upper bounds, we have that

$$
R\left((z-h)^{+}\right) \geq \chi(-\infty, z) .
$$

Conversely, let $k$ be given. We can find a sequence of central projections $\left\{e_{n}\right\}$ of sum 1 and a sequence $\left\{\alpha_{n}\right\}$ of real numbers such that

$$
\left(\alpha_{n}-(1 / 3 k)\right) e_{n}<z e_{n}<\left(\alpha_{n}+(1 / 3 k)\right) e_{n} .
$$

For $p=1,2, \ldots$ we have that

$$
\begin{aligned}
\frac{1}{k} e_{n} \chi^{\prime} & \left(\frac{1}{k}, p\right) \chi\left(\alpha_{n}-\frac{1}{3 k}, m\right) \\
& \leq(z-h) \chi^{\prime}\left(\frac{1}{k}, p\right) \chi\left(\alpha_{n}-\frac{1}{3 k}, m\right) \\
& \leq \frac{2}{3 k} e_{n} \chi^{\prime}\left(\frac{1}{k}, p\right) \chi\left(\alpha_{n}-\frac{1}{3 k}, m\right) .
\end{aligned}
$$

This shows that

$$
e_{n} \chi^{\prime}(1 / k, p) \chi\left(\alpha_{n}-(1 / 3 k), m\right)=0
$$

for all $m$ and $p$. By taking least upper bounds, we get

$$
e_{n} \chi^{\prime}(1 / k, \infty) \chi\left(\alpha_{n}-(1 / 3 k), \infty\right)=0,
$$

and consequently, that

$$
e_{n} \chi^{\prime}(1 / k, \infty) \leq e_{n} \chi\left(-\infty, \alpha_{n}-(1 / 3 k)\right] \leq e_{n} \chi(-\infty, z) .
$$

Summing over $n$, we get the inequality

$$
\chi^{\prime}(0,-\infty) \leq \chi(-\infty, z)
$$

which is the reverse of the inequality found in the last paragraph.

From the preceding proposition we see that the usual properties of spectral resolutions are true for the central spectral resolutions $\chi(-\infty, z)(h)=\chi(-\infty, z)$ on $\widetilde{Z}$. In particular, we note the properties:

(1) $\chi(-\infty, z)+\chi[z]+\chi(z, \infty)=1$;

(2) $h \chi(-\infty, z) \leq z \chi(-\infty, z)$;

(3) $h \chi(z, \infty) \geq z \chi(z, \infty)$; and

(4) $h \chi[z]=z \chi[z]$

Here we let equation (1) define $\chi[z]$. From (4) we see that all subprojections of $\chi[z]$ are in $\{h\}^{\prime \prime} \vee Z$. In addition $\chi(-\infty, z)$ has the 
usual continuity properties

(5) $\operatorname{lub}\{\chi(-\infty, z) \mid z<x\}=\chi(-\infty, x)$

as well as translation properties

(6) $\chi(-\infty, w z)(w h)=\chi(-\infty, z)(h)$, for every $w>0$ affiliated with $Z$ and

(7) $\chi(-\infty, z)(x+h)=\chi(-\infty, z-x)(h)$ for $x$ in $\tilde{Z}$. Here (6) arises from the relation

$$
\begin{aligned}
\chi(-\infty, w z)(w h) & =R\left((w z-w h)^{+}\right)=R\left(w(z-h)^{+}\right) \\
& =R\left((z-h)^{+}\right)=\chi(-\infty, z)(h)
\end{aligned}
$$

while (7) follows from the relation

$$
R\left((z-(x+h))^{+}\right)=R\left(((z-x)-h)^{+}\right) .
$$

We now recall some facts on the essential central spectrum from [4] and [14]. Let $R$ be a properly infinite semifinite algebra with center $Z$ and let $J$ be the ideal in $R$ generated by the finite projections of $R$. Let $\Omega$ be the maximal ideal space of $Z$. For $\omega \in \Omega$, let $J(\omega)$ be the ideal of $R$ generated by $J$ and $\omega$. Then for every selfadjoint element $h$ in $R$, the set $Z-\sigma^{e}(h)$ of all $z \in Z$ such that $z^{\wedge}(\omega)$ is in the spectrum of $h$ modulo $J(\omega)$ for every $\omega \in \Omega$ is called the essential central spectrum of $h$. Here $z^{\wedge}$ is the Gelfand transform of $z$. The essential central spectrum is nonempty.

Proposition 4.3. Let $z$ be in the essential central spectrum of the selfadjoint operator $h$ in the properly infinite semifinite von Neumann algebra $R$. Let $\chi$ be the spectral resolution of $h$. Then

$$
\chi(z-w, z+w)=\chi(-\infty, z+w) \chi(z-w, \infty) \sim 1
$$

for any $w$ in $Z$ with $w>0$.

Proof. There is a sequence of orthogonal central projections $\left\{e_{n}\right\}$ of sum 1 and sequence $\left\{\varepsilon_{n}\right\}$ of strictly positive numbers such that $\varepsilon_{n} e_{n}<w e_{n}$ for every $n=1,2, \ldots$. Let $p_{n}$ be the spectral projection of $(z-h) e_{n}$ corresponding to the interval $\left[-\varepsilon_{n}, \varepsilon_{n}\right]$. Then $p_{n} \sim e_{n}$ [4, Proposition 3.13]. But we have that

$$
p_{n} \leq \chi(z-w, z+w) e_{n}
$$

by the property (7) listed for the spectral resolutions. So we have that $\chi(z-w, z+w) \sim 1$.

We start by comparing $P\left(M_{\varphi}\right)$ to $P\left(M_{\tau}\right)$. We need the following lemma. 
LEMMA 4.4. Let $\varphi$ be a f.s.n. weight on a semifinite von Neumann algebra $R$. Let $\tau$ be a f.s.n. trace on $R$ and let $\chi$ be the spectral resolution of the Radon-Nikodym derivative of $\varphi$ with respect to $\tau$. Then

(i) if $\tau(\chi[\beta, \infty))+\varphi(\chi[\beta, \infty))=\infty$ for every $\beta>0$, then there is an element $x$ in $R$ such that $\varphi(x)=\infty$ and $\tau(x)<\infty$.

Furthermore, if $R$ has no type I factor direct summands, then the following are true:

(ii) if $\tau(\chi[\beta, \infty))+\varphi(\chi[\beta, \infty))=\infty$ for every $\beta>0$, then, for every $\gamma>0$, there is a $\varphi$-semifinite projection $p$ in $R$ majorized by $\chi[\gamma, \infty)$ such that $\varphi(p)=\infty$ and $\tau(p)<\infty$; and

(iii) if $\tau(\chi[\beta, \infty) e)+\varphi(\chi[\beta, \infty) e)=\infty$ for every $\beta>0$ and every nonzero central projection $e$, then there are orthogonal equivalent $\varphi$ semifinite projections $p$ and $q$ in $R$ such that $\varphi(p)<\infty, \varphi(q)=\infty$, and $\tau(p)=\tau(q)<\infty$.

Proof (i) and (ii). There is no loss of generality in the assumption that $\tau(\chi[\beta, \infty))=\infty$ for every $\beta>0$. Indeed, if there is a $\beta>$ 0 with $\tau(\chi[\beta, \infty))<\infty$, then there is no loss of generality in the assumption that $\beta>\gamma$. The projection $p=\chi[\beta, \infty)$ then satisfies the requirements of (i) and (ii) since it is $\varphi$-semifinite such that $\varphi(p)=$ $\infty$ and $\tau(p)<\infty$. So we assume that $\tau(\chi[\beta, \infty))=\infty$ for every $\beta>$ 0 . By induction, we can find a monotonely increasing sequence $\left\{\beta_{n}\right\}$ of real numbers with $\beta_{n} \geq 2^{n}$ and projections $p_{n} \leq \chi\left[\beta_{n}, \beta_{n+1}\right)$ with $2^{-n} \leq \tau\left(p_{n}\right)<\infty$ for every $n=1,2, \ldots$. We start the induction with $\beta_{1}>\gamma$. Suppose we have constructed $p_{1}, \ldots, p_{n}$ and $\beta_{1}, \ldots, \beta_{n+1}$. Because

$$
\operatorname{lub}_{\gamma} \tau\left(\chi\left[\beta_{n+1}, \gamma\right)\right)=\infty
$$

by hypothesis, there is a $\beta_{n+2}>\max \left\{\beta_{n+1}, 2^{n+1}\right\}$ such that $\tau\left(\chi\left[\beta_{n+1}, \beta_{n+2}\right)\right) \geq 2^{n+1}$. There is a projection $p_{n+1}$ with $p_{n+1} \leq$ $\chi\left[\beta_{n+1}, \beta_{n+2}\right)$ and $2^{-n-1} \leq \tau\left(p_{n+1}\right)<\infty$. This completes the induction step. We note we may assume that $\tau\left(p_{n}\right)=2^{-n}$ for every $n$ provided that $R$ has no type I factor direct summands. Setting $x=\sum 2^{-n} \tau\left(p_{n}\right)^{-1} p_{n}$ in the general case (respectively, $p=\sum p_{n}$ in the case that $R$ has no type I factor direct summands), we get a positive element $x$ (respectively, a $\varphi$-semifinite projection $p$ ) in $R$ such that

$$
\tau(x)=\sum 2^{-n} \tau\left(p_{n}\right)^{-1} \tau\left(p_{n}\right)=\sum 2^{-n}<\infty
$$


and

$$
\varphi(p)=\sum 2^{-n} \tau\left(p_{n}\right)^{-1} \varphi\left(p_{n}\right) \geq \sum \beta_{n} 2^{-n} \tau\left(p_{n}\right)^{-1} \tau\left(p_{n}\right)=\infty
$$

(respectively,

$$
\tau(p)=\sum \tau\left(p_{n}\right)=\sum 2^{-n}<\infty
$$

and

$$
\left.\varphi(p)=\sum \varphi\left(p_{n}\right) \geq \sum \beta_{n} \tau\left(p_{n}\right)=\infty\right)
$$

Proof (iii) Case I. First assume that $\tau(\chi[\beta, \infty) e)=\infty$ for every $\beta>0$ and every nonzero central projection $e$. Choose $\beta_{0}$ so that $\chi\left(-\infty, \beta_{0}\right) \neq 0$ and let $p$ be a nonzero projection of finite trace majorized by $\chi\left(-\infty, \beta_{0}\right)$. Then $\varphi(p) \leq \beta_{0} \tau(p)<\infty$. Using the fact that $R$ has no type I factor direct summands, we write $p$ as an infinite sum of mutually orthogonal nonzero projections $p=\sum p_{n}$. There is an increasing sequence $\beta_{n} \geq \beta_{0}$ such that $\sum \beta_{n} \tau\left(p_{n}\right)=\infty$. We now construct by induction a sequence of mutually orthogonal $\varphi$ s. projections $q_{n} \leq \chi\left[\beta_{n}, \infty\right)$ such that $p_{n} \sim q_{n}$. Suppose that we have constructed mutually orthogonal projections $q_{1}, q_{2}, \ldots, q_{n}$ and a sequence $\left\{e_{n i}\right\}_{i}$ of mutually orthogonal central projections of sum 1 and a corresponding sequence $\left\{\gamma_{n i}\right\}_{i}$ of positive numbers such that (1) $q_{j} \leq \chi\left[\beta_{j}, \infty\right)$ for $1 \leq j \leq n$; (2) $p_{j} \sim q_{j}$ for $1 \leq j \leq n$; and (3) $\left(q_{1}+\cdots+q_{n}\right) e_{n i} \leq \chi\left[0, \gamma_{n i}\right) e_{n i}$ for all $i$. We construct $q_{n+1}$, $\left\{e_{n+1 i}\right\}_{i}$, and $\left\{\gamma_{n+1 i}\right\}_{i}$ satisfying (1), (2) and (3). There is no loss of generality in the assumption that $e_{n i}=1$. Then let $\beta=\gamma_{n i}$. Then it is sufficient to find a single nonzero central projection $e$, a $\gamma>0$, and a projection $q_{n+1}$ with $q_{n+1}$ orthogonal to $q_{1}, q_{2}, \ldots, q_{n}$ and

(1) $q_{n+1} \leq \chi\left[\beta_{n+1}, \infty\right)$;

(2) $e p_{n+1} \sim q_{n+1}$; and

(3) $\left(q_{1}+\cdots+q_{n}+q_{n+1}\right) e \leq \chi[0, \gamma) e$.

Indeed, a maximal set of nonzero mutually orthogonal central projections $e$ satisfying the foregoing properties will have sum 1. To find $q_{n+1}$ let $\Phi$ be an operator valued trace on $R$. We have that

$$
q_{1}+\cdots+q_{n} \leq \chi[0, \beta),
$$

for some $\beta>0$ due to (3) and the assumption that $e_{n i}=1$. Let $\delta=\beta_{n+1} \vee \beta$. We have that $\chi[\delta, \infty) \sim 1$. Since $\operatorname{lub}\{\chi[\delta, \gamma) \mid$ $\delta<\gamma\}=\chi[\delta, \infty)$, there is a nonzero central projection $e$ and a $\gamma>\delta$ with $e \Phi(\chi[\delta, \gamma)) \geq e \Phi\left(p_{n+1}\right)$. This means that $e p_{n+1}$ is equivalent to a subprojection $q_{n+1}$ of the projection $e \chi[\delta, \gamma)$. Then we have 
that

(1) $q_{n+1} \leq e \chi[\delta, \gamma) \leq e \chi\left[\beta_{n+1}, \infty\right)$;

(2) $e p_{n+1} \sim q_{n+1}$; and

(3) $\left(q_{1}+\cdots+q_{n}+q_{n+1}\right) e \leq \chi[0, \beta) e+e \chi[\delta, \gamma) \leq e \chi[0, \gamma)$.

This completes the induction step.

We notice that each projection $q_{n} e_{n i}$ is in $M_{\varphi}$ since

$$
\varphi\left(q_{n} e_{n i}\right) \leq \varphi\left(\chi\left[0, \beta_{n i}\right] q_{n}\right) \leq \beta_{n i} \tau\left(q_{n}\right)=\beta_{n i} \tau\left(p_{n}\right)<\infty .
$$

Thus, the projections $q_{n}=\sum_{i} q_{n} e_{n i}$ and $q=\sum_{n} q_{n}$ are $\varphi$-semifinite due to Proposition 2.4(i). We also have that

$$
p=\sum p_{n} \sim \sum q_{n}=q
$$

and

$$
\varphi(q)=\sum \varphi\left(q_{n}\right) \geq \sum \beta_{n} \tau\left(p_{n}\right)=\infty .
$$

This completes the proof of the first case.

Proof (iii) Case II. Now assume that $\tau\left(\chi\left[\beta_{0}, \infty\right) e\right)<\infty$ for some $\beta_{0}>0$ and some nonzero central projection $e$. Then we must have that $\varphi\left(\chi\left[\beta_{0}, \infty\right) f\right)=\infty$ for every nonzero central projection $f$ majorized by $e$. Since $\varphi$ is a semifinite faithful normal weight on the semifinite algebra $R_{e}$ without type I factor direct summands, there is no loss of generality in the assumption that $\tau\left(\chi\left[\beta_{0}, \infty\right)\right)<\infty$ and that $\varphi(\chi[\beta, \infty) e)=\infty$ for every nonzero central projection $e$ and every $\beta \geq \beta_{0}$. By using the normality of $\varphi$ and $\tau$, we can find two monotonely increasing sequences $\beta_{0}<\beta_{n}<\gamma_{n} \leq \beta_{n+1}$ such that $\tau\left(\chi\left[\beta_{n}, \infty\right)\right) \leq 2^{-n}$ and $\varphi\left(\chi\left[\beta_{n}, \gamma_{n}\right)\right) \geq 1$. Let $q_{n}=\chi\left[\beta_{n}, \gamma_{n}\right)$ and let $q=\sum q_{n}$. We also note that $q$ is a $\varphi$-s. projection (Proposition 2.4(ii)). By construction, we have that $q \notin M_{\varphi}$ but $q \in M_{\tau}$. Since $\chi\left[\beta_{0}, \infty\right)$ is a finite projection, we have that $\chi\left(-\infty, \beta_{0}\right) \sim 1$. Because $\chi\left(-\infty, \beta_{0}\right)$ is a $\varphi$-s. projection, we can find a $\varphi$-s. projection $p \leq$ $\chi\left(-\infty, \beta_{0}\right)$ with $p \sim q$ (Lemma 2.5). Then $\varphi(p) \leq \beta_{0} \tau(p)<\infty$.

The next lemma treats the lower part of the spectral resolution of the Radon-Nikodym derivative in a manner similar to Lemma 4.4.

LEMMA 4.5. Let $\varphi$ be a f.s.n. weight on a semifinite von Neumann algebra $R$. Let $\tau$ be a f.s.n. trace on $R$ and let $\chi$ be the spectral resolution of the Radon-Nikodym derivative of $\varphi$ with respect to $\tau$. Then

(i) if $\tau(\chi(-\infty, \alpha))=\infty$ for every $\alpha>0$, then, for $\varepsilon>0$, there is a positive element $x$ in $R$ such that $\varphi(x)<\varepsilon$ and $\tau(x)=\infty$; and 
(ii) if $\tau(\chi(-\infty, \alpha) e)=\infty$ for every $\alpha>0$ and every nonzero central projection $e$, then, given $\varepsilon>0$, there is a projection $p$ in $R$ such that $\varphi(p)<\varepsilon$ and $p \sim 1-p \sim 1$.

Furthermore,

(iii) if $R$ has no type I factor direct summands, and if $\tau(\chi(-\infty, \alpha))$ $=\infty$ for every $\alpha>0$, then, for $\varepsilon>0$ and every $\gamma>0$, there is $a$ projection $p$ in $R$ majorized by $\chi(-\infty, \gamma)$ such that $\varphi(p)<\varepsilon$ and $\tau(p)=\infty$.

Proof (i) and (iii). Let $\left\{\alpha_{n}\right\}$ be a strictly decreasing sequence of positive real numbers such that $\alpha_{1}=\gamma$ and such that $\sum \alpha_{n}<\infty$. By induction we can find a decreasing sequence $\left\{\beta_{n}\right\}$ of positive numbers such that $\beta_{n} \leq \alpha_{n}$ and a sequence of projections $p_{n}$ with $p_{n} \leq$ $\chi\left(\beta_{n+1}, \beta_{n}\right]$ such that $\alpha_{n} \leq \tau\left(p_{n}\right)<\infty$. As in Lemma 4.4, we can find a projection $p_{n}$ with $p_{n} \leq \chi\left(\beta_{n+1}, \beta_{n}\right]$ such that $\alpha_{n}=\tau\left(p_{n}\right)$ provided $R$ has no type I factor direct summands. Setting $x=\sum \tau\left(p_{n}\right)^{-1} p_{n}$ in the general case (respectively, $p=\sum p_{n}$ in the case that $R$ has no type I factor direct summands), we get a positive element $x$ (respectively, a $\varphi$-semifinite projection $p$ by Proposition 2.4(ii)) in $R$ such that

$$
\tau(x)=\sum \tau\left(p_{n}\right)^{-1} \tau\left(p_{n}\right)=\infty
$$

and

$$
\varphi(x)=\sum \beta_{n} \tau\left(p_{n}\right)^{-1} \tau\left(p_{n}\right) \leq \sum \alpha_{n}<\infty
$$

(respectively,

and

$$
\tau(p)=\sum \tau\left(p_{n}\right)=\infty
$$

$$
\left.\varphi(p) \leq \sum \beta_{n} \tau\left(p_{n}\right)<\infty\right)
$$

Proof (ii). The hypothesis is equivalent to the statement that $\chi(-\infty, \alpha) \sim 1$ for every $\alpha>0$. This means in particular that $R$ is properly infinite. Let $r_{0}$ be a finite projection in $R$ such that $\tau\left(r_{0}\right)=\delta$ for some $\delta>0$. Let $\left\{\alpha_{n}\right\}$ be a monotonely decreasing sequence of strictly positive real numbers such that $\sum \delta \alpha_{n}<\varepsilon$. We construct a sequence $\left\{r_{n}\right\}$ of mutually orthogonal projections such that

$$
r_{0} \sim r_{n} \leq \chi\left(-\infty, \alpha_{n}\right)
$$

for every $n=1,2, \ldots$. Suppose that we have constructed the finite set $r_{1}, \ldots, r_{n}$; we construct $r_{n+1}$. Let $r=r_{1}+\cdots+r_{n}$. We see that

$$
\chi\left(-\infty, \alpha_{n+1}\right)-R\left(\chi\left[\left(-\infty, \alpha_{n+1}\right) r\right)\right) \sim 1
$$


because $\chi\left(-\infty, \alpha_{n+1}\right) \sim 1$ by hypothesis and because the range projection $R\left(\chi\left(-\infty, \alpha_{n+1}\right) r\right)$ of $\chi\left(-\infty, \alpha_{n+1}\right) r$ is a finite projection since the range projection is equivalent to a subprojection of $r$. We can therefore find a projection $r_{n+1}$ with

$$
r_{0} \sim r_{n+1} \leq \chi\left(-\infty, \alpha_{n+1}\right)-R\left(\chi\left(-\infty, \alpha_{n+1}\right) r\right) .
$$

Then we have that

$$
r_{n+1} r=r_{n+1} \chi\left(-\infty, \alpha_{n+1}\right) r=r_{n+1}\left(R\left(\chi\left(-\infty, \alpha_{n+1}\right) r\right)\right) r=0 .
$$

Thus, we have completed the induction step. We now have a sequence of mutually orthogonal projections $\left\{r_{n}\right\}$ with $r_{0} \sim r_{n}$ and with $r_{n} \leq$ $\chi\left(-\infty, \alpha_{n}\right)$ for every $n=1,2, \ldots$. Setting $p=\sum r_{n}$, we get that

$$
\varphi(p)=\sum \varphi\left(r_{n}\right)=\sum \tau\left(h r_{n}\right) \leq \sum \alpha_{n} \tau\left(r_{n}\right)=\sum \alpha_{n} \tau\left(r_{0}\right)<\infty .
$$

We also have that $p \sim c\left(r_{0}\right)$.

Now let $\left\{p_{n}\right\}$ be a maximal set of nonzero projections in $R$ with orthogonal central supports such that $\varphi\left(p_{n}\right)<\varepsilon$ and $p_{n} \sim c\left(p_{n}\right)$. The material in the previous paragraph shows that $\sum p_{n}=1$. Since $R p_{n}$ is properly infinite, we can decompose each $p_{n}$ into $2^{n}$ mutually equivalent orthogonal projections $\left\{p_{n k}\right\}_{k}$ of sum $p_{n}$. One of the projections $p_{n k}$ satisfies $\varphi\left(p_{n k}\right)<\varepsilon / 2^{n}$ and $p_{n k} \sim c\left(p_{n k}\right)-p_{n k} \sim$ $c\left(p_{n k}\right)$. So we may assume that $\varphi\left(p_{n}\right)<\varepsilon / 2^{n}$ and $p_{n} \sim c\left(p_{n}\right)-p_{n} \sim$ $c\left(p_{n}\right)$. Therefore, the projection $p=\sum p_{n}$ satisfies $\varphi(p)<\varepsilon$ and $p \sim 1-p \sim 1$.

If the ideals of definition of a f.s.n. weight and a f.s.n. trace are related by inclusion, then the weight and the trace bound one another up to a finite functional.

Proposition 4.6. Let $R$ be a semifinite von Neumann algebra. Let $\varphi$ be a f.s.n. weight on $R$ and let $\tau$ be a f.s.n. trace on $R$. Then a(i) $M_{\varphi} \subset M_{\tau}$ if and only if a(ii) there is an $\alpha>0$ and a positive normal functional $\omega_{1}$ such that $\alpha \tau-\omega_{1} \leq \varphi$; and $\mathrm{b}(\mathrm{i}) M_{\tau} \subset M_{\varphi}$ if and only if $\mathrm{b}(\mathrm{ii})$ there is $a \quad \beta>0$ and a positive normal functional $\omega_{2}$ such that $\varphi \leq \beta \tau+\omega_{2}$.

Proof. We only need to show that (i) implies (ii). We use Lemmas 4.4(i) and 4.5(i). First we show that a(i) implies a(ii). Let $\chi$ be the spectral resolution of the Radon-Nikodym derivative of $\varphi$ with respect to $\tau$. By Lemma 4.5(i), there is an $\alpha>0$ such that 
$\tau(\chi(-\infty, \alpha))<\infty$ for some $\alpha>0$; otherwise, there would be a positive element $x$ in $R$ with $\varphi(x)<\infty$ and $\tau(x)=\infty$ contrary to a(i). But then we have that

$$
\alpha \tau \leq \varphi+\alpha \tau(\chi(-\infty, \alpha)) .
$$

Letting $\omega_{1}=\alpha \tau(\chi(-\infty, \alpha) \cdot)$, we see that $\omega_{1}$ is finite and $\alpha \tau-\omega_{1} \leq$ $\varphi$.

The implication $b(\mathbf{i})$ implies $b(i i)$ follows in a similar manner from Lemma 4.4(i).

When the algebra $R$ has no type I factor direct summands, we can rephrase Proposition 4.6 in terms of the projection lattices.

Proposition 4.7. Let $R$ be a semifinite von Neumann algebra with no type I factor direct summands. Let $\varphi$ be a f.s.n. on $R$ and let $\tau$ be a f.s.n. trace on $R$. Then $M_{\varphi} \subset M_{\tau}$ if and only if $P\left(M_{\varphi}\right) \subset P\left(M_{\tau}\right)$ and $M_{\tau} \subset M_{\varphi}$ if and only if $P\left(M_{\tau}\right) \subset P\left(M_{\varphi}\right)$.

Proof. First let $P\left(M_{\varphi}\right) \subset P\left(M_{\tau}\right)$. Let $\chi$ be the spectral resolution of the Radon-Nikodym derivative of $\varphi$ with respect to $\tau$. By Lemma 4.5(ii), there is an $\alpha>0$ such that $\tau(\chi(-\infty, \alpha))<\infty$ for some $\alpha>0$; otherwise, there would be a projection $p$ with $\varphi(p)<\infty$ and $\tau(p)=\infty$ contrary to the assumption that $P\left(M_{\varphi}\right) \subset P\left(M_{\tau}\right)$. As in Proposition 4.5(a), there is a normal functional $\omega$ on $R$ such that

$$
\alpha \tau-\omega \leq \varphi \text {. }
$$

This shows that $M_{\varphi} \subset M_{\tau}$.

The proof of the second part of Proposition 4.6 is similar. Here Lemma 4.4(ii) is used instead of Lemma 4.5(ii).

In the remainder of this section we consider f.s.n. weights $\varphi$ such that $P\left(M_{\varphi}\right)$ is a lattice. Here we need to separate two cases: with type I factor summands and without such summands. We first prove a lemma that is used in both cases.

LEMMA 4.8. Let $\varphi$ be a f.s.n. weight on a properly infinite semifinite von Neumann algebra $R$ such that $P\left(M_{\varphi}\right)$ is a lattice. If $\left\{e_{n}\right\}$ is a sequence of mutually orthogonal central projections such that $\varphi\left(e_{n}\right)<$ $\infty$ for every $n$, then $\sum \varphi\left(e_{n}\right)<\infty$.

Proof. Let $\chi$ be the spectral resolution of the Radon-Nikodym derivative of $\varphi$ with respect to a f.s.n. trace $\tau$ on $R$. Then the 
projection $\chi[\beta, \infty) e_{n}$ has finite trace for every $\beta>0$ because

$$
\beta \tau\left(\chi[\beta, \infty) e_{n}\right) \leq \varphi\left(\chi[\beta, \infty) e_{n}\right) \leq \varphi\left(e_{n}\right)<\infty .
$$

Because $R$ is properly infinite $\tau(\chi(-\infty, \alpha) e)=\infty$ for every $\alpha>0$ and every nonzero central projection $e$ majorized by $e_{n}$. Lemma 4.5(ii) applied to $R e_{n}$ implies the existence of a projection $p_{n} \leq$ $e_{n}$ with $\varphi\left(p_{n}\right) \leq 2^{-n}$ such that $p_{n} \sim e_{n}-p_{n} \sim e_{n}$. However, the projection $p=\sum p_{n}$ now satisfies $\varphi(p) \leq 1$ and

$$
p \sim \sum\left(e_{n}-p_{n}\right) \sim \sum e_{n}-p \sim \sum e_{n} .
$$

So we have obtained two orthogonal equivalent $\varphi$-semifinite projections $p$ and $\sum e_{n}-p$ (cf. Proposition 2.4(i) and (ii)). Now the characterization of the lattice property of $P\left(M_{\varphi}\right)$ in Proposition 3.2 forces

$$
\varphi\left(\sum e_{n}-p\right)<\infty
$$

which taken together with $\varphi(p)<\infty$ forces

$$
\sum \varphi\left(e_{n}\right)<\infty
$$

Now we consider the first of the two cases.

THeOREM 4.9. Let $R$ be a properly infinite semifinite von Neumann algebra without type I factor direct summands. Let $\varphi$ be a f.s.n. weight on $R$. Then $P\left(M_{\varphi}\right)$ is a lattice if and only if there is a central projection $e$ and a f.s.n. trace $\tau$ on $R$ such that

(i) $\varphi$ restricted to $R_{e}$ is a functional; and

(ii) $P\left(M_{\tau}\right)=P\left(M_{\varphi}\right)$ on $R_{(1-e)}$.

Proof. Let $\varphi$ be a f.s.n. weight on $R$ such that $P\left(M_{\varphi}\right)$ is a lattice. First let $\left\{e_{n}\right\}$ be a maximal set of mutually orthogonal nonzero central projections such that $\varphi\left(e_{n}\right)<\infty$. Setting $e=\sum e_{n}$, we get a central projection $e$ such that $\varphi$ is a functional on $R_{e}$ (Lemma 4.8) and such that $\varphi(f)=\infty$ for every nonzero central projection $f$ in $R_{(1-e)}$. We note that the finite projections of $\varphi \mid R_{(1-e)}$ is still a lattice. So by reducing to the f.s.n. weight on the properly infinite semifinite algebra $R_{(1-e)}$ with no type I factor direct summands, there is no loss of generality in the assumption $\varphi(f)=\infty$ for every central projection $f$ in $R$.

We construct a f.s.n. trace $\tau$ with $P\left(M_{\tau}\right)=P\left(M_{\varphi}\right)$. Let $\tau$ be any f.s.n. trace on $R$ and let $h$ be the Radon-Nikodym derivative of $\varphi$ 
with respect to $\tau$. Let $\chi$ be the spectral resolution of $h$. We modify $\tau$ by multiplying it by an element affiliated with the center constructed from $h$ in order to get the desired trace.

First, let $\left\{e_{n}\right\}$ be a maximal set of nonzero mutually orthogonal central projections in $R$ such that for each $e_{n}$ there is a $\beta_{n}>0$ with

$$
\tau\left(\chi\left[\beta_{n}, \infty\right) e_{n}\right)+\varphi\left(\chi\left[\beta_{n}, \infty\right) e_{n}\right)<\infty .
$$

We must have that $\sum e_{n}=1$; otherwise, we must have that every nonzero projection $e$ majorized by the nonzero projectional $1-\sum e_{n}$ satisfies

$$
\tau(\chi[\beta, \infty) e)+\varphi(\chi[\beta, \infty) e)=\infty
$$

for every $\beta>0$. Then there would be two orthogonal equivalent projections $p$ and $q$ in $R$ such that $\varphi(p)<\infty, \varphi(q)=\infty$ (Lemma 4.4(iii)) and $P\left(M_{\varphi}\right)$ would not be a lattice (Proposition 3.2). So we must have that $\sum e_{n}=1$.

Second, let $\left\{f_{n}\right\}$ be a maximal set of nonzero orthogonal central projections in $R$ such that for each $f_{n}$ there is an $\alpha_{n}>0$ with

$$
\tau\left(\chi\left(-\infty, \alpha_{n}\right) f_{n}\right)<\infty .
$$

By the same reasoning as the preceding paragraph we have that $\sum f_{n}=$ 1. Here we use Lemma 4.5(ii).

Now by combining the sets $\left\{e_{n}\right\}$ and $\left\{f_{n}\right\}$ into a single set, we may assume that there is a sequence of mutually orthogonal central projections $\left\{e_{n}\right\}$ of sum 1 and two sequences $\left\{\alpha_{n}\right\}$ and $\left\{\beta_{n}\right\}$ of real numbers with $0<\alpha_{n}<\beta_{n}$ such that

$$
\tau\left(\chi\left(-\infty, \alpha_{n}\right) e_{n}\right)+\tau\left(\chi\left[\beta_{n}, \infty\right) e_{n}\right)+\varphi\left(\chi\left[\beta_{n}, \infty\right) e_{n}\right)<\infty
$$

for every $n=1,2, \ldots$. We can also write

$$
\begin{aligned}
\tau\left(\chi\left(-\infty, \alpha_{n}\right) e_{n}\right) & +\varphi\left(\chi\left(-\infty, \alpha_{n}\right) e_{n}\right) \\
& +\tau\left(\chi\left[\beta_{n}, \infty\right) e_{n}\right)+\varphi\left(\chi\left[\beta_{n}, \infty\right) e_{n}\right)<\infty .
\end{aligned}
$$

We have that $\tau(p)<\infty$ if and only if $\varphi(p)<\infty$ for every projection $p \leq e_{n}$. In fact, we have that $\tau(p)<\infty$ (respectively, $\varphi(p)<\infty$ ) if and only if $\tau\left(p \chi\left[\alpha_{n}, \beta_{n}\right) e_{n}\right)<\infty$ (respectively, $\varphi\left(p \chi\left[\alpha_{n}, \beta_{n}\right) e_{n}\right)<$ $\infty)$ so that the relation

$$
\alpha_{n} \tau\left(\chi\left[\alpha_{n}, \beta_{n}\right) p e_{n}\right) \leq \varphi\left(\chi\left[\alpha_{n}, \beta_{n}\right) p e_{n}\right) \leq \beta_{n} \tau\left(\left(\chi\left[\alpha_{n}, \beta_{n}\right) p e_{n}\right)\right)
$$

shows that $\tau$ and $\varphi$ mutually bound each other on projections majorized by $e_{n}$. 
Now we have that each $h_{n}$ given by

$$
h_{n}=h\left(1-\left(\chi\left(-\infty, \alpha_{n}\right)+\chi\left[\beta_{n}, \infty\right)\right)\right) e_{n}
$$

is a bounded positive operator on the properly infinite von Neumann algebra $R_{e_{n}}$. Let $z_{n}$ be an element in the essential central spectrum of $h_{n}$. We have that

$$
\alpha_{n} e_{n} \leq z_{n} \leq \beta_{n} e_{n}
$$

since

$$
\alpha_{n} e_{n} \leq h_{n} \leq \beta_{n} e_{n}
$$

modulo the ideal generated by finite projections in $R_{e_{n}}$. Thus, for any projection $p$, the number $\tau\left(z_{n} p\right)$ is finite if and only if $\varphi\left(z_{n} p\right)$ is finite.

Now we show that the trace

$$
\tau_{0}(x)=\sum \tau\left(z_{n} x\right)
$$

is the desired trace. From this point to the end of the proof we do not use the fact that $R$ has no type I factor direct summands. We present an argument based entirely on the fact that $\alpha_{n} e_{n} \leq z_{n} \leq \beta_{n} e_{n}$. So we must show that $P\left(M_{\tau_{0}}\right)=P\left(M_{\varphi}\right)$. First let $p$ be an arbitrary projection in $R$ with $\tau\left(p_{n}\right)+\varphi\left(p_{n}\right)<\infty$ for every $n=1,2, \ldots$. Here $p_{n}=p e_{n}$. Let $\left\{\varepsilon_{n}\right\}$ be a sequence of positive real numbers such that

$$
\sum \varepsilon_{n} \tau\left(p_{n}\right)<\infty
$$

Then we have that

$$
\chi\left(z_{n}-\varepsilon_{n} e_{n}, z_{n}+\varepsilon_{n} e_{n}\right) \sim e_{n}
$$

by Proposition 4.3. The range projection

$$
r_{n}=R\left(\chi\left(z_{n}-\varepsilon_{n} e_{n}, z_{n}+\varepsilon_{n} e_{n}\right) p_{n}\right)
$$

is a finite projection and satisfies

$$
\varphi\left(r_{n}\right) \leq \beta_{n} \tau\left(p_{n}\right)<\infty
$$

So we have that

$$
r_{n}^{\prime}=\chi\left(z_{n}-\varepsilon_{n} e_{n}, z_{n}+\varepsilon_{n} e_{n}\right)-r_{n}
$$

is a $\varphi$-s. projection (Proposition 2.4(iii)) equivalent to $e_{n}$. Then we can find a $\varphi$-s. projection $q_{n}$ with

$$
p_{n} \sim q_{n} \leq r_{n}^{\prime}
$$


due to Lemma 2.5. Actually, the projection $q_{n}$ is in $M_{\varphi}$. We see that $p_{n}$ is orthogonal to $q_{n}$ since

$q_{n} p_{n}=q_{n} \chi\left(z_{n}-\varepsilon_{n} e_{n}, z_{n}+\varepsilon_{n} e_{n}\right) p_{n}=q_{n} r_{n} \chi\left(z_{n}-\varepsilon_{n} e_{n}, z_{n}+\varepsilon_{n} e_{n}\right) p=0$.

We also see that

$$
\tau\left(\left(z_{n}+\varepsilon_{n}\right) q_{n}\right)-\varepsilon_{n} \tau\left(q_{n}\right) \leq \tau_{0}\left(p_{n}\right)=\tau_{0}\left(q_{n}\right) \leq \tau\left(\left(z_{n}-\varepsilon_{n}\right) q_{n}\right)+\varepsilon_{n} \tau\left(q_{n}\right)
$$

since

$$
\varphi\left(q_{n}\right)=\tau\left(h_{n} \chi\left(z_{n}-\varepsilon_{n} e_{n}, z_{n}+\varepsilon_{n} e_{n}\right) q_{n}\right) \leq \tau\left(\left(z_{n}+e_{n}\right) q_{n}\right)
$$

and likewise that

$$
\tau\left(\left(z_{n}-\varepsilon\right) q_{n}\right) \leq \varphi\left(q_{n}\right) .
$$

Setting $\sum q_{n}=q$, we get a $\varphi$-s. projection $q$ with $p q=0$ and $q \sim p$ such that

$$
\varphi(q)-\sum \varepsilon_{n} \tau\left(q_{n}\right) \leq \tau_{0}(p)=\tau_{0}(q) \leq \varphi(q)+\sum \varepsilon_{n} \tau\left(q_{n}\right) .
$$

Now we use the material in the last paragraph to complete the proof. Suppose that $p \in P\left(M_{\varphi}\right)$. Let $p_{n}=p e_{n}$. Then we have $\tau\left(p_{n}\right)+\varphi\left(p_{n}\right)<\infty$ for every $n=1,2, \ldots$ due to the first part of the proof. This means that the projection $q$ constructed in the previous paragraph is in $P\left(M_{\varphi}\right)$; otherwise, the set $P\left(M_{\varphi}\right)$ would not be a lattice by Proposition 3.2. Since $\sum \varepsilon_{n} \tau\left(q_{n}\right)<\infty$, we have that $p$ is in $P\left(M_{\tau_{0}}\right)$. So we have that $P\left(M_{\varphi}\right) \subset P\left(M_{\tau_{0}}\right)$.

Conversely, suppose that $p \in P\left(M_{\tau_{0}}\right)$. Again we have $\tau_{0}\left(p_{n}\right)<\infty$ for every $n=1,2, \ldots$. This means that $\tau\left(p_{n}\right)<\infty$ for every $n$ and thus that $\varphi\left(p_{n}\right)<\infty$ for every $n$. Now by the previous part of the proof we find a $\varphi$-s. projection $q$ with $p q=0, p \sim q$, and $\sum \varepsilon_{n} \tau\left(q_{n}\right)<\infty$ such that

$$
\varphi(q)-\sum \varepsilon_{n} \tau\left(q_{n}\right) \leq \tau_{0}(p)=\tau_{0}(q) \leq \varphi(q)+\sum \varepsilon_{n} \tau\left(q_{n}\right) .
$$

So we get that $q$ is in $P\left(M_{\varphi}\right)$, and consequently, $p$ is in $P\left(M_{\varphi}\right)$ by Proposition 3.2. This means that $P\left(M_{\tau_{0}}\right) \subset P\left(M_{\varphi}\right)$.

Now we complete the analysis of the properly infinite semifinite case.

THEOREM 4.10. Let $R=\sum \bigoplus R_{n}$ where $R_{n}$ are type $\mathrm{I}_{\infty}$ factors and let $\varphi$ be f.s.n. weight on $R$. Then $P\left(M_{\varphi}\right)$ is a lattice if and only if the identity is the sum of three orthogonal central projections $e, f$ and $g$ such

(i) $\varphi$ is a finite functional on $R_{e}$; 

(ii) $P\left(M_{\varphi}\right) \subset P\left(M_{\mathrm{Tr}}\right)$ on $R_{f}$; and
(iii) $P\left(M_{\varphi}\right)=P\left(M_{\tau}\right)$ on $R_{g}$.

Here $\operatorname{Tr}=\sum \bigoplus \operatorname{tr}$ where $\operatorname{tr}$ is the canonical trace on a type $\mathrm{I}_{\infty}$ factor and $\tau$ is f.s.n. trace on $R_{g}$.

Proof. We show that $P\left(M_{\varphi}\right) \subset P\left(M_{\mathrm{Tr}}\right)$ on $R$ implies $P\left(M_{\varphi}\right)$ is a lattice. Let $e_{n}$ be the central projection of $R$ which is the identity on $R_{n}$. Let $p, q \in P\left(M_{\varphi}\right)$; then $p \vee q \in P\left(M_{\mathrm{Tr}}\right)$. Since $p \vee q$ is a sum of minimal projections, each with trace one, we see that $(p \vee q) e_{n}=0$ for all but a finite number of indices. For these indices

$$
(p \vee q) e_{n} \leq \gamma_{n}(p+q) e_{n}
$$

for some $\gamma_{n}>0$ because $p e_{n}$ and $q e_{n}$ are finite dimensional projections. But then

$$
\varphi(p \vee q) \leq \max \left\{\gamma_{n}\right\} \varphi(p+q)<\infty,
$$

whence $p \vee q \in P\left(M_{\varphi}\right)$. Thus $P\left(M_{\varphi}\right)$ is a lattice.

Now assume that $P\left(M_{\varphi}\right)$ is a lattice. Let $\chi$ be the Radon-Nikodym derivative $h$ of $\varphi$ with respect to $\operatorname{Tr}$ and as before let $e_{n}$ be the central projection of $R$ such that $R e_{n}=R_{n}$. First let $S_{1}$ be the set

$$
S_{1}=\left\{n \mid \varphi\left(e_{n}\right)<\infty\right\} \text {. }
$$

Then we must have that

$$
\sum\left\{\varphi\left(e_{n}\right) \mid n \in S_{1}\right\}<\infty
$$

by Lemma 4.8 , i.e., $\varphi$ is a finite functional on $R_{e}$ where $e=$ $\sum\left\{e_{n} \mid n \in S_{1}\right\}$.

Now we consider $\varphi$ on the direct sum $\sum \bigoplus\left\{R_{n} \mid n \notin S_{1}\right\}$. Again there is no loss of generality in the assumption that

$$
\sum\left\{e_{n} \mid n \notin S_{1}\right\}=1 \text {. }
$$

First we show that, for every $n$, there is $\alpha_{n}>0$ such that $\chi\left(0, \alpha_{n}\right) e_{n}=$ 0 . We have that $\varphi\left(e_{n}\right)=\infty$. If $\operatorname{tr}\left(\chi(-\infty, \alpha) e_{n}\right)=\infty$ for all $\alpha>0$, then we could again find a projection $p \leq e_{n}$ with $\varphi(p)<\infty$ and $p \sim e_{n}-p \sim e_{n}$ by Lemma 4.5(ii). This also contradicts Proposition 3.2. So we must have that $\operatorname{tr}\left(\chi(-\infty, \alpha) e_{n}\right)<\infty$ for some $\alpha>0$. We have that

$$
\operatorname{glb}\left\{\operatorname{tr}\left(\chi(-\infty, \alpha) e_{n}\right) \mid \alpha>0\right\}=0
$$

since $\varphi$ is a f.s.n. when restricted to $R e_{n}$. Because $\operatorname{tr}\left(\chi(-\infty, \alpha) e_{n}\right)$ is integer valued, we must have that $\operatorname{tr}\left(\chi(-\infty, \alpha) e_{n}\right)=0$ for some $\alpha>0$. Let

$$
\alpha_{n}=\operatorname{lub}\left\{\alpha \mid \operatorname{tr}\left(\chi(-\infty, \alpha) e_{n}\right)=0\right\}
$$


We have that $\chi\left(-\infty, \alpha_{n}\right) e_{n}=0$ while $\chi\left(-\infty, \alpha_{n}\right] e_{n} \neq 0$. The latter is due to the fact that $\alpha \rightarrow \operatorname{tr}\left(\chi(-\infty, \alpha) e_{n}\right)$ is integer valued and left continuous.

Now let

$$
S=\left\{n \mid \chi[1, \infty) e_{n} \sim e_{n}\right\}
$$

Suppose that $n \in S$. We show that

$$
\gamma=\operatorname{glb}\left\{\alpha_{n} \mid n \in S\right\}>0 .
$$

We obtain a contradiction if $\gamma=0$. By passing to a subset of $S$, we may assume that $\sum \alpha_{n}<\infty$. Then there are two infinite orthogonal sequences of one dimensional projections $\left\{p_{n}\right\}$ and $\left\{q_{n}\right\}$ such that

$$
p_{n} \leq \chi\left(-\infty, \alpha_{n}\right] e_{n}
$$

and

$$
q_{n} \leq \chi\left(1, \beta_{n}\right] e_{n}
$$

for every $n$. Here $\beta_{n}$ is some number $\beta_{n} \geq 1$. However, this would give two equivalent $\varphi$-s. projections $p=\sum p_{m}$ and $q=\sum q_{m}$ with

$$
\varphi(p) \leq \sum \alpha_{n} \operatorname{tr}\left(p_{n}\right)=\sum \alpha_{n}<\infty
$$

and

$$
\varphi(q)>\sum \beta_{n} \operatorname{tr}\left(q_{n}\right)=\sum \beta_{n}=\infty .
$$

Again this would contradict Proposition 3.2. So we must have $\gamma>0$. Now let $f=\sum\left\{e_{n} \mid n \in S\right\}$. Then the weight $\varphi$ restricted to $R_{f}$ is a f.s.n. weight with

$$
\varphi(p) \geq \gamma \operatorname{Tr}(p \chi[\gamma, \infty))=\gamma \operatorname{Tr}(p f(\chi(-\infty \cdot \gamma)+\chi[\gamma, \infty)))=\gamma \operatorname{Tr}(p f)
$$

for every projection $p$ majorized by $f$. This means that $P\left(M_{\varphi}\right) f \subset$ $P\left(M_{\mathrm{Tr}}\right)$.

Now we consider the final set of indices, the complement of $S$ and $S_{1}$. We have that $\chi[1, \infty) e_{n}$ is a finite projection in $R e_{n}$; otherwise, the projection $\chi[1, \infty) e_{n}$ would be infinite and $n$ would be in $S$. Since $\operatorname{lub}_{\beta} \operatorname{tr}\left(\chi\left[\beta_{n}, \infty\right) e_{n}\right)=0$, there is a $\beta_{n}>0$ with $\chi\left[\beta_{n}, \infty\right) e_{n}=$ 0 . Thus, there are numbers $0<\alpha_{n}<\beta_{n}$ such that $\chi\left(-\infty, \alpha_{n}\right) e_{n}=$ $\chi[\beta, \infty) e_{n}=0$. Now we can finish the proof in the same way we finished the proof of Theorem 4.9. Let $\delta_{n}$ be in the essential spectrum of the bounded operator $h e_{n}$. Let $\tau$ be the trace $\sum \bigoplus \delta_{n} \operatorname{tr}$. Then we have that $P\left(M_{\varphi}\right)=P\left(M_{\tau}\right)$.

REMARK 4.11. In the case of type $\mathrm{I}_{\infty}$ factors the inclusion $P\left(M_{\varphi}\right) \subset$ $P\left(M_{\mathrm{tr}}\right)$ does not in general imply that $P\left(M_{\varphi}\right)=P\left(M_{\mathrm{tr}}\right)$. In fact, if 
$h$ is the Radon-Nikodym derivative of $\varphi$ with respect to $\mathrm{tr}$, then $P\left(M_{\varphi}\right)=P\left(M_{\mathrm{tr}}\right)$ implies that $h$ is bounded. Indeed, if there were a vector $\xi \notin D\left(h^{1 / 2}\right)$, then the rank 1 projection on the subspace generated by $\zeta$ would be in $P\left(M_{\mathrm{tr}}\right)$ but not in $P\left(M_{\varphi}\right)$. So $P\left(M_{\varphi}\right)=$ $P\left(M_{\mathrm{tr}}\right)$ implies that $h$ is defined on the whole Hilbert space and thus that $h$ is bounded.

On the other hand $P\left(M_{\varphi}\right)=P\left(M_{\mathrm{tr}}\right)$ does not in general imply that $M_{\varphi}=M_{\mathrm{Tr}}$. For example, the weight $\varphi=\sum \bigoplus n \operatorname{tr}$ satisfies $P\left(M_{\varphi}\right)=P\left(M_{\mathrm{Tr}}\right)$ but $M_{\varphi} \neq M_{\mathrm{Tr}}$.

5. Finite algebras. To treat finite algebras we need to develop additional functional calculus for central intervals.

Proposition 5.1. Let $R$ be a finite von Neumann algebra with center $Z$, let $\Phi$ be the canonical center valued trace on $R$, let $h \eta R$, $h=h^{*} \geq 0$ with null space $N(h)=0$ and let

$$
z=\operatorname{lub}\{x \eta Z \mid x>0, \Phi(\chi(-\infty, x)) \leq(1 / 2) 1\} .
$$

Then $z \eta Z, z>0$ and

$$
\Phi(\chi(-\infty, z)) \leq(1 / 2) 1 \text { and } \Phi(\chi(z, \infty)) \leq(1 / 2) 1 \text {. }
$$

Proof. Let $\left\{e_{n}\right\}$ (respectively, $\left\{f_{n}\right\}$ ) be a maximal family of nonzero mutually orthogonal central projections such that there are strictly positive numbers $\left\{\alpha_{n}\right\}$ (respectively, $\left.\left\{\beta_{n}\right\}\right)$ with $\Phi\left(\chi\left(-\infty, \alpha_{n}\right) e_{n}\right) \leq$ $2^{-1} e_{n}$ (respectively, $\left.\Phi\left(\chi\left(-\infty, \beta_{n}\right) f_{n}\right) \geq(2 / 3) f_{n}\right)$. Then we must have that $\sum e_{n}=1$ (respectively, $\sum f_{n}=1$ ) since the limit in the strong operator topology of $\{\Phi(\chi(-\infty, \alpha))\}$ as $\alpha$ goes to 0 (respectively, $\infty$ ) is 0 (respectively, 1 ). We have that

$$
\sum \alpha_{n} e_{n} \leq \sum \beta_{n} f_{n} \text {. }
$$

Then the set of all sums $\sum \alpha_{n} e_{n}$ where $\left\{e_{n}\right\}$ is a sequence of mutually orthogonal projections of sum 1 and $\left\{\alpha_{n}\right\}$ is a sequence of strictly positive numbers such that $\sum \Phi\left(\chi\left(-\infty, \alpha_{n}\right) e_{n}\right) \leq 2^{-1} 1$ is upward by the ordering described in the introduction to $\S 4$. In addition the set of all sums is bounded above by the sums $\sum \beta_{n} f_{n}$. Thus, we have that

$$
z=\operatorname{lub}\left\{\sum \alpha_{n} e_{n} \mid \sum e_{n}=1 \text { and } \sum \Phi\left(\chi\left(-\infty, \alpha_{n}\right) e_{n}\right) \leq 2^{-1}\right\}
$$

is a positive selfadjoint element affiliated with $Z$ such that

$$
\Phi(\chi(-\infty, z)) \leq 2^{-1} 1 \text {. }
$$


Now let $x$ and $y$ be positive selfadjoint elements affiliated with $Z$ such that $z<x<y$. Then we have that

$$
\Phi(\chi(-\infty, x)) \geq 2^{-1} 1
$$

and

$$
\Phi(\chi(y, \infty)) \leq 1-\Phi(\chi(-\infty, x)) \leq 2^{-1} 1 .
$$

Taking the least upper bound of $\Phi(\chi(y, \infty))$ for all $y>z$, we get

$$
\Phi(\chi(z, \infty)) \leq 2^{-1} 1 \text {. }
$$

Now let $\varphi$ be a f.s.n. weight on the finite von Neumann algebra $R$. Let $\tau_{1}$ and $\tau_{2}$ be two f.n.s. traces on $R$ and let $h_{1}$ and $h_{2}$ be the Radon-Nikodym derivatives of $\varphi$ with respect to $\tau_{1}$ and $\tau_{2}$ respectively. Let $\chi_{1}$ and $\chi_{2}$ be the spectral resolutions of $h_{1}$ and $h_{2}$ respectively. Let $z_{1}$ and $z_{2}$ be the operators associated to $h_{1}$ and $h_{2}$ respectively by Proposition 5.1, viz.,

$$
z_{i}=\operatorname{lub}\left\{x \eta Z \mid x>0, \Phi\left(\chi_{i}(-\infty, x)\right) \leq(1 / 2) 1\right\} .
$$

There is a $w \eta Z^{+}$with $w>0$ such that

$$
\tau_{1}(w x)=\tau_{2}(x)
$$

for all $x \in R^{+}$. So we have that $w h_{2}=h_{1}$. We have already seen in Property 6 of the central spectral resolution given in $\S 4$ that

$$
\begin{aligned}
\chi_{1}(-\infty, z) & =\chi(-\infty, z)\left(h_{1}\right) \\
& =\chi(-\infty, w z)\left(w h_{1}\right)=\chi(-\infty, w z)\left(h_{2}\right)=\chi_{2}(-\infty, w z) .
\end{aligned}
$$

Taking into account the definition of $z_{1}$ and $z_{2}$ as least upper bounds, we get that $z_{1}=w z_{2}$. This means that

$$
\tau_{1}\left(z_{1} x\right)=\tau_{1}\left(w z_{2} x\right)=\tau_{2}\left(z_{2} x\right)
$$

for all $x$ in $R^{+}$.

Now the following definition makes sense.

Definition 5.2. Let $R$ be a finite von Neumann algebra and $\varphi$ be a f.s.n. weight. Let $\tau$ be a f.s.n. trace on $R$ and let

$$
z=\operatorname{lub}\{x \eta Z \mid x>0, \Phi(\chi(-\infty, x)) \leq(1 / 2) 1\}
$$

where $\Phi$ is the canonical operator valued trace on $R$ and $\chi$ is the spectral resolution of the Radon-Nikodym derivative $h$ of $\varphi$ with respect to $\tau$. Then the trace $\tau_{\varphi}=\tau \cdot z$ is called canonical trace associated with the weight $\varphi$.

The canonical trace balances at 1 the spectral resolution of the Radon-Nikodym derivative. 
Proposition 5.3. Let $\varphi$ be a f.s.n. weight on the finite von Neumann algebra $R$ with center $Z$, let $\tau_{\varphi}$ be the canonical trace associated with $\varphi$, and let $h$ be the Radon-Nikodym derivative of $\varphi$ with respect to $\tau_{\varphi}$. Then

$$
1=\operatorname{lub}\{x \eta Z \mid x>0, \Phi(\chi(-\infty, x)(h)) \leq(1 / 2) 1\},
$$

where $\Phi$ is the canonical operator valued trace on $R$.

Proof. Setting

$$
z=\sup \{x \eta Z \mid x>0, \Phi(\chi(-\infty, x)(h)) \leq(1 / 2) 1\}
$$

we have that $\tau_{\varphi}=\tau_{\varphi} \cdot z$, and consequently, we have that $z=1$.

Proposition 5.4. Let $R$ be a finite von Neumann algebra, let $\varphi$ be a f.s.n. weight on $R$, let $\tau_{\varphi}$ be the canonical trace associated with $\varphi$, and $h$ be the Radon-Nikodym derivative of $\varphi$ with respect to $\tau_{\varphi}$. Then there are equivalent projections $r_{-}$and $r_{+}$in $R^{\varphi}$ such that (i) $h r_{-} \leq r_{-}$, (ii) $r_{+} \leq h r_{+}$; and (ii) $h\left(1-\left(r_{-}+r_{+}\right)\right)=1-\left(r_{-}+r_{+}\right)$.

Proof. By taking a central decomposition, we may assume that $R$ is either a type $\mathrm{II}_{1}$ or a type $\mathrm{I}_{n}$ algebra. First assume that $R$ is a type $\mathrm{II}_{1}$ algebra. Then we have that $h \chi(-\infty, 1) \leq \chi(-\infty, 1)$, $\chi(1, \infty) \leq h \chi(1, \infty)$, and $h \chi[1]=\chi[1]$. Now we have that

$$
\Phi(\chi(-\infty, 1)) \leq(1 / 2) 1 \leq 1-\Phi(\chi(1, \infty))=\Phi(\chi(-\infty, 1))+\Phi(\chi[1]) .
$$

Since $R$ is a continuous algebra, there is a subprojection $p$ of $\chi[1]$ such that $\Phi(\chi[0,1)+p)=1$. Setting $r_{-}=\chi(-\infty, 1)+p$ and $r_{+}=$ $1-r_{-}$, we get two projections $r_{-}$and $r_{+}$in $R^{\varphi}$ due to the fact that every subprojection of $\chi[1]$ is in $R^{\varphi}$. We also have that

$$
h r_{-} \leq h(\chi(-\infty, 1)+\chi[1]) r_{-} \leq r_{-}
$$

and

$$
h r_{+}=h(\chi(1, \infty)+(\chi[1]-p)) r_{+} \leq r_{+} .
$$

Since the condition $\Phi\left(r_{-}\right)=\Phi\left(r_{+}\right)$implies that $r_{-} \sim r_{+}$, we have constructed the desired projections in the type $\mathrm{II}_{1}$ case. Note that $r_{+}+r_{-}=1$ in this case.

If $R$ is a type $I_{n}$ algebra, we may assume that by passing to a central summand that $\chi(-\infty, 1), \chi[1]$, and $\chi(1, \infty)$ are equal respectively to the sum of $n_{1}, n_{2}$ and $n_{3}$ mutually orthogonal maximal abelian 
projections. Then $n_{1} /\left(n_{1}+n_{2}+n_{3}\right) \leq 1 / 2$ while $n_{3} /\left(n_{1}+n_{2}+n_{3}\right) \leq$ $1 / 2$. Now it is clear that one can find $r_{-}$and $r_{+}$using the fact that two maximal abelian projections are equivalent.

It is instructive to consider a type $\mathrm{I}_{n}$ factor algebra $M_{n}$. Let $\operatorname{tr}$ be the trace $\operatorname{tr}\left(\left(a_{i j}\right)\right)=\sum a_{i i}$. We may assume that the Radon-Nikodym derivative $h$ of the weight $\varphi$ with respect to tr is the diagonal matrix

$$
h=\operatorname{diag}\left(a_{1}, \ldots, a_{n}\right)
$$

with $0<a_{1} \leq a_{2} \leq \cdots \leq a_{n}$. Then a decomposition of the identity satisfying the requirements of Proposition 5.4 is

$$
r_{-}=\operatorname{diag}(1, \ldots, 1,0, \ldots, 0),
$$

and

$$
r_{+}=\operatorname{diag}(0, \ldots, 0,1, \ldots, 1),
$$

where there are [n/2] ones in both $r_{-}$and $r_{+}$. Here [n/2] is the integer part of $n / 2$. We note that the decomposition of Proposition 5.4 is not unique. However, the canonical trace is unique and is given by

$$
\tau_{\varphi}=a_{[(n+1) / 2]} \operatorname{tr}
$$

We can now discuss the lattice properties of $P\left(M_{\varphi}\right)$ for a finite algebra.

THeOREM 5.5. Let $R$ be a finite algebra, let $\varphi$ be a f.s.n. weight and let $\tau_{\varphi}$ be the associated canonical trace. Then $P\left(M_{\varphi}\right)$ is a lattice if and only if $P\left(M_{\varphi}\right)=P\left(M_{\tau_{\varphi}}\right)$.

Proof. We can prove the necessity in the separate cases (i) $R$ is a direct sum of type I factors and (ii) $R$ has no type I factor direct summands. We have already presented a proof for the direct sum of type I factors in [7]. We sketch the proof again for the sake of completeness.

First let $R$ be the direct sum of matrix algebras

$$
R=\sum_{n} \bigoplus M_{k(n)}
$$

We have already seen that the canonical trace for $\varphi$ is

$$
\tau_{\varphi}=\sum_{n} \bigoplus a_{n, m(n)} \operatorname{tr}
$$


where $m(n)=[(k(n)+1) / 2]$. Given any projection $p=\sum_{n} \oplus p_{n}$ in $R$, we define

$r_{n}=\operatorname{diag}(1, \ldots, 1,0, \ldots, 0)$ and $s_{n}=\operatorname{diag}(0, \ldots, 0,1, \ldots, 1)$, both with $\min \left\{\operatorname{tr}\left(p_{n}\right),[k(n) / 2]\right\}$ ones, and we set

$$
r=\sum_{n} \bigoplus r_{n} \text { and } s=\sum_{n} \bigoplus s_{n}
$$

Here we shall again use the assumption that $h_{n}$ are diagonal matrices. The projections $r$ and $s$ are orthogonal equivalent projections. By definition of $r_{n}$ and $s_{n}$ and by the monotonicity of $\left\{a_{n, j}\right\}$, we have

$$
\operatorname{tr}\left(r_{n}\right) \leq \operatorname{tr}\left(p_{n}\right) \leq 2 \operatorname{tr}\left(r_{n}\right)
$$

and

$$
\operatorname{tr}\left(h_{n} r_{n}\right) \leq a_{n, m(n)} \operatorname{tr}\left(r_{n}\right) \leq \operatorname{tr}\left(h_{n} s_{n}\right)
$$

We have that

$$
\operatorname{tr}\left(h_{n} r_{n}\right) \leq \sum_{j=1}^{\operatorname{tr}\left(p_{n}\right)} a_{n, j} \leq \sum_{j=1}^{k(n)} a_{n, m}\left(p_{n}\right)_{j j}=\operatorname{tr}\left(h_{n} p_{n}\right)
$$

and likewise that $\operatorname{tr}\left(h_{n} p_{n}\right) \leq 3 \operatorname{tr}\left(h_{n} s_{n}\right)$. Therefore, we obtain the inequalities

$$
\begin{aligned}
\varphi(r) & \leq \tau_{\varphi}(r) \leq \varphi(s), \\
\tau_{\varphi}(r) & \leq \tau_{\varphi}(p) \leq 3 \tau_{\varphi}(r), \\
\varphi(r) & \leq \varphi(p) \leq 3 \varphi(s) .
\end{aligned}
$$

Now assume that $P\left(M_{\varphi}\right) \neq P\left(M_{\tau_{\varphi}}\right)$. We shall obtain a contradiction from the preceding inequalities by showing the existence of two orthogonal $\varphi$-semifinite projections, one of which is in $M_{\varphi}$ and one of which is not. This is impossible on account of Proposition 3.2. On the one hand, if $p \in M_{\varphi}$ but $p \notin M_{\tau_{\varphi}}$, then $r \in M_{\varphi}, r \notin M_{\tau_{\varphi}}$ and hence $s \notin M_{\varphi}$. On the other hand, if $p \in M_{\tau_{\varphi}}$ but $p \notin M_{\varphi}$, then $s \notin M_{\varphi}$ and hence $r \in M_{\varphi}$. In either case, we have two equivalent orthogonal projections $r$ and $s$, one of which is in $M_{\varphi}$ and one of which is not. But every projection in a finite von Neumann algebra is $\varphi$-semifinite (Proposition 2.3). Thus, we have obtained a contradiction. Hence, we conclude that $P\left(M_{\varphi}\right)=P\left(M_{\tau_{\varphi}}\right)$ whenever $P\left(M_{\varphi}\right)$ is a lattice.

Now assume that $R$ has no type I factor summands and let $r_{-}$and $r_{+}$be the equivalent, orthogonal projections in $R^{\varphi}$ given by Proposition 5.4 applied to the canonical trace $\tau_{\varphi}$ with respect to $\varphi$. First 
we show that $P\left(M_{\varphi}\right) \not \subset P\left(M_{\tau_{\varphi}}\right)$ leads to a contradiction. Indeed, let $\chi$ be the spectral resolution of the Radon-Nikodym derivative of $\varphi$ with respect to $\tau_{\varphi}$. We have already seen that $\tau_{\varphi}(\chi(-\infty, \alpha))<\infty$ for some $\alpha>0$ implies that $P\left(M_{\varphi}\right) \subset P\left(M_{\tau_{\varphi}}\right)$ since

$$
\tau_{\varphi}(x) \leq \alpha^{-1} \varphi(x)+\tau_{\varphi}(\chi(-\infty, \alpha) x)
$$

whenever $x \geq 0$. So if $P\left(M_{\varphi}\right) \not \subset P\left(M_{\tau_{\varphi}}\right)$ were true, we must have that $\tau_{\varphi}(\chi(-\infty, \alpha))=\infty$ for all $\alpha>0$. Thus, there would be a projection $p \leq \chi(-\infty, 1)=r_{-}$such that $\varphi(p)<\infty$ and $\tau_{\varphi}(p)=\infty$ (Lemma 4.5(iii)). Since $r_{-} \sim r_{+}$, we could find a $\varphi$-semifinite projection $q \leq$ $r_{+} \leq \chi[1, \infty)$ which is equivalent to $p$ due to Lemma 2.5. However, we would then have that

$$
\varphi(q)=\tau_{\varphi}(h q) \geq \tau_{\varphi}(q)=\tau_{\varphi}(p)=\infty .
$$

This would now mean that $P\left(M_{\varphi}\right)$ is not a lattice due to Proposition 3.2. On the other hand, if $P\left(M_{\tau_{\varphi}}\right) \not \subset P\left(M_{\varphi}\right)$, then we also get a contradiction. We would have that

$$
\tau_{\varphi}(\chi[\beta, \infty))+\varphi(\chi[\beta, \infty))=\infty
$$

for all $\beta>0$ and so we could find a $\varphi$-s. projection $p \leq \chi(1, \infty) \leq$ $r_{+}$, such that $\tau_{\varphi}(p)<\infty$ and $\varphi(p)=\infty$ (Lemma 4.4(ii)). But then there is a projection $q \leq r_{-}$with $q \sim p$ because $r_{-} \sim r_{+}$(Proposition 5.4). Since

$$
\varphi(q)=\tau_{\varphi}(h q) \leq \tau_{\varphi}(q)=\tau_{\varphi}(p)<\infty,
$$

we would conclude, again by Proposition 3.2, that $P\left(M_{\varphi}\right)$ is not a lattice. Thus, we must have that $P\left(M_{\tau_{\varphi}}\right) \subset P\left(M_{\varphi}\right)$. Combining this with the previous inclusion found in the first part of the proof, we get that $P\left(M_{\tau_{\varphi}}\right)=P\left(M_{\varphi}\right)$ whenever $P\left(M_{\varphi}\right)$ is a lattice.

Now we can compute $I(\varphi)$ for finite algebras.

THEOREM 5.6. Let $\varphi$ be a f.s.n. weight on a finite algebra $R$ and let $h$ be the Radon-Nikodym derivative of $\varphi$ with respect to the canonical trace $\tau_{\varphi}$ of $\varphi$. Let $r_{-}$and $r_{+}$be any projections in $R^{\varphi}$ with $r_{-} \sim r_{+}$ in $R$ such that (i) $h r_{-} \leq r_{-}$, (ii) $r_{+} \leq h r_{+}$, and (iii) $h\left(1-r_{-}-r_{+}\right)=$ $1-r_{-}-r_{+}=r_{0}$. Then $I(\varphi)=2 \varphi\left(r_{-}\right)+\varphi\left(r_{0}\right)$.

Proof. We note that projections $r_{-}$and $r_{+}$exist by Proposition 5.4 but are not necessarily unique since the piece of $\chi[1]$ is not determined. Then there is a sequence $\left\{e_{n}\right\}$ of mutually orthogonal central 
projections of sum 1 such that $\tau_{\varphi}\left(e_{n}\right)<\infty$ for every $n=1,2, \ldots$. We have that $\tau_{\varphi} \cdot e_{n}$ is the canonical trace associated with the weight $\varphi \cdot e_{n}$ on $R_{e_{n}}$. We also have that

$$
\sum I\left(\varphi \cdot e_{n}\right)=I(\varphi)
$$

and

$$
\sum\left(2 \varphi\left(e_{n} r_{-}\right)+\varphi\left(e_{n} r_{0}\right)\right)=2 \varphi\left(r_{-}\right)+\varphi\left(r_{0}\right) .
$$

Thus, there is no loss of generality in the assumption that $\tau_{\varphi}$ is a finite trace. Then we have that

$$
\varphi\left(r_{-}\right)=\tau_{\varphi}\left(h r_{-}\right) \leq \tau_{\varphi}\left(r_{-}\right)<\infty
$$

so that $\varphi\left(r_{-} \cdot\right)$ is a finite functional. Therefore, for every projection $p$, we can decompose $\varphi(p)$ as

$$
\begin{aligned}
\varphi(p) & =\varphi\left(r_{-}\right)-\varphi\left(r_{-}(1-p)\right)+\varphi\left(\left(1-r_{-}\right) p\right) \\
& \geq \varphi\left(r_{-}\right)-\tau_{\varphi}\left(r_{-}(1-p)\right)+\tau_{\varphi}\left(\left(1-r_{-}\right) p\right) \\
& =\varphi\left(r_{-}\right)+\tau_{\varphi}(p)-\tau_{\varphi}\left(r_{-}\right) .
\end{aligned}
$$

Now we take now a second projection $q$ such that $p \vee q=1$. We have that $1-p \sim q-p \wedge q$ by the Parallelogram Law. In defining $I(\varphi)$, we have already remarked that there is no loss of generality in the assumption that $p \wedge q=0$. Using this assumption, the Parallelogram Law for $p$ and $q$ becomes $1-p \sim q$. Applying the inequality in the preceding paragraph to both $p$ and $q$ and adding the results, we get

$$
\begin{aligned}
I(\varphi) & \geq \varphi(p+q) \geq 2 \varphi\left(r_{-}\right)+\tau_{\varphi}(p+q)-2 \tau_{\varphi}\left(r_{-}\right) \\
& =2 \varphi\left(r_{-}\right)+\tau_{\varphi}(1)-\tau_{\varphi}\left(r_{-}+r_{+}\right) \\
& \geq 2 \varphi\left(r_{-}\right)+\tau_{\varphi}\left(r_{0}\right) \\
& \geq 2 \varphi\left(r_{-}\right)+\varphi\left(r_{0}\right) .
\end{aligned}
$$

To prove the reverse inequality, we apply Lemma 3.1 to the pair of orthogonal equivalent projections $r_{-}$and $r_{+}$. Notice that $r_{+}$is $\varphi$-semifinite since it is in $R^{\varphi}$ (Proposition 2.4(ii)). Thus, for every $\varepsilon>0$, there is a projection $q$ such that $r_{-} \vee q=r_{-}+r_{+}$and $\varphi(q)<$ $\varphi\left(r_{-}\right)+\varepsilon$. Because $\left(r_{-}+r_{0}\right) \vee q=1$, we get

$$
I(\varphi) \leq \varphi\left(r_{-}+r_{0}+q\right) \leq 2 \varphi\left(r_{-}\right)+\varphi\left(r_{0}\right)+\varepsilon .
$$

Since $\varepsilon>0$ is arbitrary, we have that

$$
I(\varphi) \leq 2 \varphi\left(r_{-}\right)+\varphi\left(r_{0}\right) .
$$


In particular we see that $2 \varphi\left(r_{-}\right)+\varphi\left(r_{0}\right)$ does not depend on the choice of $r_{-}$and $r_{+}$with the properties (i)-(iii) of Proposition 5.4.

6. Calculation of $I(\varphi)$ and $J(\varphi)$ for semifinite algebras. We have already calculated $I(\varphi)$ for finite algebras. We also know that $I(\varphi)=$ $\infty$ if the identity is not the supremum of two projections in $M_{\varphi}$. We complete the remaining case in the next theorem.

First we need to extend the notion of essential central spectrum to an unbounded selfadjoint operator $h$ affiliated with a von Neumann algebra $R$ with center $Z$. The selfadjoint operator $z$ affiliated with $z$ will be said to be in the essential central spectrum of $h$ if

$$
\chi(z-w, z+w)(h) \sim 1
$$

for every $w>0$ affiliated with $z$. This corresponds to the behavior of the essential central spectrum for bounded operators (cf. Proposition 4.3).

THEOREM 6.1. Let $\varphi$ be a f.s.n. weight on a properly infinite semifnite von Neumann algebra $R$ and let $h$ be the Radon-Nikodym derivative of $\varphi$ with respect to a f.s.n. trace $\tau$. Then the following are equivalent:

(i) $0 \in Z-\sigma^{e}(h)$;

(ii) $I(\varphi)=0$; and

(iii) $I(\varphi)<\infty$, i.e., the identity is the supremum of two projections in $M_{\varphi}$.

Proof (i) implies (ii). Let $0 \in Z-\sigma^{e}(h)$; then $\chi(0, \alpha) \sim 1$ for all $\alpha>0$. This means that, given $\varepsilon>0$, there is a projection $p$ in $R$ with $\varphi(p)<\varepsilon$ and $p \sim 1-p \sim 1$ (Lemma 4.5(ii)). We can find a projection $q$ with $\varphi(q)<\varepsilon$ and $p \vee q=p+(1-p)=1$ (Lemma 3.1) because $1-p$ is $\varphi$-semifinite (Proposition 2.4(iii)). This means that $I(\varphi)<2 \varepsilon$. Since $\varepsilon>0$ is arbitrary, we have that $I(\varphi)=0$.

Proof (iii) implies (i). Suppose that there are two projections $p$ and $q$ in $M_{\varphi}$ with $p \vee q=1$. We get a contradiction if $\chi(-\infty, \alpha) e$ is a finite projection for some nonzero central projection $e$ and some $\alpha>0$. In fact, if $\chi(-\infty, \alpha) e$ is a finite projection, we have that

$$
\alpha \tau(\chi[\alpha, \infty) p e) \leq \tau(h \chi[\alpha, \infty) p e) \leq \varphi(p e)<\infty .
$$

This means that $\chi[\alpha, \infty) p e$ and consequently

$$
p e=\chi(-\infty, \alpha) p e+\chi(\alpha, \infty) p e
$$


are finite projections. Likewise, the projection $q e$ is finite so that pe $\vee q e=e$ is finite contrary to our assumption that $R$ is properly infinite. Thus $\chi(-\infty, \alpha) \sim 1$ for all $\alpha>0$.

Let $\varphi$ be a n.s.f. weight on the von Neumann algebra $R$. Using Theorem 6.1, we compute the parameter $J(\varphi)$. Recall that we have already shown in Proposition 3.5 that $J(\varphi)=\infty$ whenever $R$ has a nonzero type III direct summand. So we can restrict our attention to semifinite algebras.

Let $h$ be a positive faithful selfadjoint element affiliated with the von Neumann algebra $R$ with center $Z$. Let $\chi$ be the spectral resolution of $h$. On the one hand, there is a set $\left\{e_{n}\right\}$ of central projections of sum 1 and a sequence $\left\{\alpha_{n}\right\}$ of positive numbers such that $\sum \chi\left(-\infty, \alpha_{n}\right) e_{n}=0$. Thus, the set of elements in $Z$ given by

$$
\{z \in Z \mid \chi(-\infty, z)=0\}
$$

is upward directed and

$$
v_{h}=\operatorname{lub}\{z \in Z \mid \chi(-\infty, z)=0\}
$$

is a positive selfadjoint element affiliated with $Z$.

On the other hand, there is a maximal set $\left\{f_{n}\right\}$ of nonzero orthogonal central projections and a set $\left\{\beta_{n}\right\}$ of numbers such that $\sum \chi\left(\beta_{n}, \infty\right) f_{n}=0$. Let $\sum f_{n}=f_{h}$ and let

$$
w_{h}=\operatorname{glb}\left\{z \eta Z f_{h} \mid \chi(z, \infty)=0\right\}+\infty\left(1-f_{h}\right) .
$$

Since $w f_{h}=\operatorname{glb}\left\{\chi(z, \infty) \mid z \eta Z f_{h}\right\}$ is a positive selfadjoint element affiliated with $Z f_{h}$ due to the fact that $h$ is faithful and positive selfadjoint, the element $w$ is in $\widehat{Z}^{+}$(cf. [2]).

Proposition 6.2. Let $h$ be a faithful positive selfadjoint element affiliated with the von Neumann algebra $R$. Let $\chi$ be the spectral resolution of $h$. Let

$$
v_{h}=\operatorname{lub}\{z \in Z \mid \chi(-\infty, z)=0\}
$$

and

$$
w_{h}=\operatorname{glb}\left\{z \eta Z f_{h} \mid \chi(z, \infty)=0\right\}+\infty\left(1-f_{h}\right)
$$

where $\left(1-f_{h}\right)$ is the largest projection $f$ in $Z$ such that $\chi(\alpha, \infty)$ has central support $f$ for every $\alpha$. Then

(i) $\chi(-\infty, z)$ has central support 1 for every $z$ affiliated with $Z$ such that $z>v_{h}$; 
(ii) $\chi(z, \infty)$ has central support 1 for every $z$ affiliated with $Z$ such that $z<w_{h}$; and

(iii) $v_{h} \leq h \leq w_{h}$.

Proof (i). Suppose that $z$ is affiliated with $Z$ with $v_{h}<z$. Then there is a sequence $\left\{e_{n}\right\}$ of central projections of sum 1 and sequences of numbers $\left\{\alpha_{n}\right\}$ and $\left\{\beta_{n}\right\}$ such that

$$
v_{h} e_{n}<\alpha_{n} e_{n}<z e_{n}<\beta_{n} e_{n} .
$$

We must have that $\chi\left(-\infty, \alpha_{n}\right) e_{n}$ has central support $e_{n}$ by the definition of $v_{h}$. Thus, the projection $\sum \chi\left(-\infty, \alpha_{n}\right) e_{n}$ and consequently the projection $\chi(-\infty, z)$ has central support 1 .

Proof (ii). Same as (i).

Proof (iii). Let $\left\{e_{n}\right\}$ be an orthogonal sequence of central projections of sum 1 and let $\left\{\alpha_{n}\right\}$ be a sequence of numbers with

$$
\sum \chi\left(-\infty, \alpha_{n}\right) e_{n}=0 \text {. }
$$

Then we have that, for all $m_{n}>\alpha_{n}$,

$$
\alpha_{n} \chi\left(-\infty, m_{n}\right) e_{n} \leq h \chi\left(-\infty, m_{n}\right) e_{n} .
$$

Then for all finite sums we have that

$$
\sum \alpha_{n} \chi\left(-\infty, m_{n}\right) e_{n}=\sum h \chi\left(-\infty, m_{n}\right) e_{n} .
$$

Hence, we have that

$$
\sum \alpha_{n} e_{n} \leq h
$$

and finally, that

$$
v_{h} \leq h
$$

Now let $\left\{e_{n}\right\}$ be an orthogonal sequence of central projections of sum $f_{h}$ and let $\left\{\beta_{n}\right\}$ be a sequence of numbers with $\sum \chi\left(\beta_{n}, \infty\right) e_{n}$ $=0$. Then we have that

$$
h \chi\left(j_{n}, k_{n}\right) e_{n} \leq \beta_{n} \chi\left(j_{n}, k_{n}\right) e_{n}
$$

for all $j_{n}<\beta_{n}<k_{n}$. So we have that

$$
h \chi\left(j_{n}, k_{n}\right) e_{n} \leq w_{h} e_{n}
$$

as a relation for bounded operators and

$$
h f_{h} \leq w_{h} f_{h}
$$


by taking least upper bounds. Since $h\left(1-f_{h}\right) \leq \infty\left(1-f_{h}\right)$, we get

$$
h \leq w_{h} \text {. }
$$

Definition 6.3. Let $\varphi$ be a f.s.n. weight on the semifinite von Neumann algebra $R$ with center $Z$, let $\tau$ be a f.s.n. trace on $R$, and let $h$ be the Radon-Nikodym derivative of $\varphi$ with respect to $\tau$. Let $\chi$ be the spectral resolution of $h$ and let

$$
v=v_{h}=\operatorname{lub}\{z \in Z \mid \chi(-\infty, z)=0\}
$$

and

$$
w=w_{h}=\operatorname{glb}\left\{z \eta Z f_{h} \mid \chi(z, \infty)=0\right\}+\infty\left(1-f_{h}\right)
$$

where $\left(1-f_{h}\right)$ is the largest projection $f$ in $Z$ such that $\chi(\alpha, \infty)$ has central support $f$ for every $\alpha$. Then the central size of $\varphi$ is the number

$$
\gamma=\gamma_{\varphi}= \begin{cases}\left\|v^{-1} w\right\| & \text { if } v^{-1} w \text { is bounded } \\ \infty & \text { otherwise. }\end{cases}
$$

Notice that $v$ and $w$ do not depend on the choice of the trace. In fact, if $\tau^{\prime}$ is a second n.s.f. trace on $R$, then there is an $x>0$ affiliated with $Z$ such that

$$
\tau=\tau^{\prime} \cdot x
$$

The Radon-Nikodym derivative of $\varphi$ with respect to $\tau^{\prime}$ is $x h$. Then we have that $\chi(-\infty, z)(h)=0$ if and only if $\chi(-\infty, \chi z)(x h)=0$ (cf. $\S 4$, Property 6) so that $\chi v_{h}=v_{x h}$. Similar reasoning gives $\chi w_{h}=$ $w_{x h}$.

THEOREM 6.4. If $R$ is a semifinite algebra, let $\varphi$ be a f.s.n. weight on $R$ and let $\gamma=\gamma_{\varphi}$ be the central size of $\varphi$; then

$$
J(\varphi)-\frac{1}{2}(1+\gamma)
$$

Proof. Let us first prove that $J(\varphi) \leq \frac{1}{2}(1+\gamma)$. Clearly we need to consider only the case that $\gamma \neq \infty$. Since

$$
\tau^{\prime} \leq \varphi \leq \gamma \tau^{\prime}
$$

where $\tau^{\prime}=\tau(v \cdot)$, we have $M_{\varphi}=M_{\tau^{\prime}}$. Let $p$ and $q$ be nonzero projections in $M_{\varphi}$; then $r=p \vee q$ is in $M_{\tau^{\prime}}$. Hence $R_{r}$ is a finite algebra and so the restriction $\varphi^{\prime}$ of $\varphi$ to $R_{r}$ is finite and normal and faithful. Thus, by Proposition 5.4 applied to $R_{r}$, we can find 
a decomposition of $r=r_{-}+r_{0}+r_{+}$into three mutually orthogonal projections such that $r_{-} \sim r_{+}$and

$$
\varphi^{\prime}(p+q)=\varphi(p+q) \geq I\left(\varphi^{\prime}\right) \geq 2 \varphi^{\prime}\left(r_{-}\right)+\varphi^{\prime}\left(r_{0}\right) \geq 2 \varphi\left(r_{-}\right)+\varphi\left(r_{0}\right) .
$$

Then we get

$$
\begin{aligned}
\frac{\varphi(p \vee q)}{\varphi(p+q)} & \leq \frac{\varphi\left(r_{-}\right)+\varphi\left(r_{0}\right)+\varphi\left(r_{+}\right)}{2 \varphi\left(r_{-}\right)+\varphi\left(r_{0}\right)} \\
& \leq \frac{1}{2}+\frac{2 \tau\left(w r_{+}\right)+\varphi\left(r_{0}\right)}{2 \tau\left(v r_{-}\right)+\varphi\left(r_{0}\right)} \leq \frac{1}{2}+\frac{2 \gamma \tau\left(v r_{+}\right)+\varphi\left(r_{0}\right)}{2 \tau\left(v r_{+}\right)+\varphi\left(r_{0}\right)}
\end{aligned}
$$

so that

$$
\frac{\varphi(p \vee q)}{\varphi(p+q)} \leq \frac{1}{2}(1+\gamma)
$$

since $\gamma \geq 1$. Since $p$ and $q$ are arbitrary, we obtain

$$
J(\varphi) \leq \frac{1}{2}(1+\gamma)
$$

Now we prove the reverse inequality. First suppose that $\chi$ is the spectral resolution of $h$. Suppose that $\chi\left(\alpha, \alpha^{\prime}\right)$ and $\chi\left(\beta, \beta^{\prime}\right)$ have the same nonzero central support $e$ for some $0 \leq \alpha<\alpha^{\prime}<\beta<\beta^{\prime}$. Then there are finite equivalent $p$ and $q$ with $p \leq \chi\left(\alpha, \alpha^{\prime}\right)$ and $q \leq \chi\left(\beta, \beta^{\prime}\right)$. For every $\eta>0$, there is a projection $q_{\eta}$ such that $\varphi\left(q_{\eta}\right)<\varphi(p)+\eta$ and $q_{\eta} \vee p=p+q$. Then we have

$$
J(\varphi) \geq \frac{\varphi\left(p \vee q_{\eta}\right)}{\varphi\left(p+q_{\eta}\right)} \geq \frac{\varphi(p+q)}{2 \varphi(p)+\eta} \geq \frac{(\alpha+\beta) \tau(p)}{2 \alpha^{\prime} \tau(p)+\eta} .
$$

Since $\eta>0$ is arbitrary, we have that

$$
J(\varphi) \geq \frac{\alpha+\beta}{2 \alpha^{\prime}} .
$$

Now we consider two cases: (i) the null projection $e$ of $v$ is nonzero and (ii) $e=0$. In case (i) we have that $\gamma=\infty$. Let $\beta>0$ be any number such that $\chi(\beta, \infty) e \neq 0$. By replacing $e$ by a smaller nonzero projection if necessary, there is no loss of generality in the assumption that $e$ is a nonzero central projection such that $v e=0$ and $\chi(\beta, \infty) e$ has central support $e$. Now by the definition $v$ we have that $\chi\left(0, \alpha^{\prime}\right) e$ has central support $e$ for every $\alpha^{\prime}>0$. By the previous paragraph there are projections $p$ and $q$ with

$$
\frac{\varphi(p \vee q)}{\varphi(p+q)} \geq \frac{\beta}{2 \alpha^{\prime}}
$$

Since $\beta>0$ is fixed and $\alpha^{\prime}$ can be arbitrarily small, we get the desired relation $J(\varphi)=\infty$. 
Assume now that $e=0$. For every integer $n$ define

$$
\delta_{n}= \begin{cases}\gamma-\frac{1}{n} & \text { if } \gamma \neq \infty, \\ n & \text { if } \gamma=\infty\end{cases}
$$

If $\gamma=1$, then $v=w=h$; hence, we see that $\varphi$ is a trace and therefore $J(\varphi)=1=(1+\gamma) / 2$.

Assume, therefore, that $\gamma>1$, and choose $n$ so that also $\delta_{n}<\gamma$. Then, by the definition of $\gamma$, we can find a nonzero central projection $f$ such that $\delta_{n} f<w v^{-1} f$. For every $\varepsilon>0$, there are mutually orthogonal projections $\chi\left(\alpha, \alpha^{\prime}\right)$ and $\chi(\beta, \infty)$ with $\beta / \alpha^{\prime}>\delta$ such that

$$
c\left(\chi\left(\alpha, \alpha^{\prime}\right)\right) c(\chi(\beta, \infty)) \neq 0 .
$$

As before, we have that

$$
J(\varphi) \geq 2^{-1}\left(1+\left(\beta / \alpha^{\prime}\right)\right) \geq 2^{-1}(1+\delta) .
$$

This means that $J(\varphi) \geq(1+\gamma) / 2$ as desired.

COROLlary 6.5. The weight $\varphi$ is a trace if and only if $J(\varphi)=1$.

Proof. On the one hand, if $J(\varphi)=1$, then $R$ can have no type III direct summand (Proposition 3.5). Thus, the algebra $R$ is semifinite. Then we have that $\gamma_{\varphi}=1$ and $\varphi=\tau \cdot h$ for some injective positive selfadjoint operator with $v \leq h \leq v$ with $v \in \widehat{Z}^{+}$. This means that $h=v$ and thus that $\varphi$ is a trace. On the other hand, if $\varphi$ is a trace, then $\gamma_{\varphi}=1$ and $J(\varphi)=\left(1+\gamma_{\varphi}\right) / 2=1$.

7. The main result. We can now restate our main theorem.

THEOREM 1. Let $R$ be a $\sigma$-finite von Neumann algebra and let $\varphi$ be a f.s.n. weight on $R$. Then $P\left(M_{\varphi}\right)$ is a lattice if and only if there is a decomposition of the identity into mutually orthogonal central projections $e+f+g=1$ such that $R_{f}$ is a semifinite algebra and $R_{g}$ is a direct sum of type $\mathrm{I}_{\infty}$ factors equipped with the f.s.n. trace $\operatorname{Tr}$ (the direct sum of the canonical traces on the factors) so that

(a) $\varphi$ restricted to $R_{e}$ is a finite functional,

(b) $P\left(M_{\varphi(f .)}\right)=P\left(M_{\tau}\right)$ for some f.s.n. trace $\tau$ on $R_{f}$, and

(c) $P\left(M_{\varphi(g \cdot)}\right) \subset P\left(M_{\mathrm{Tr}}\right)$.

Proof. The proof follows from combining the statements of Proposition 3.3, Theorem 4.9, Theorem 4.10, and Theorem 5.5. Note that 
some of the type $I_{\infty}$ factors have been included in (ii) due to Theorem 4.10.

\section{REFERENCES}

[1] A. Amann and L. Zsidó, Private communication.

[2] U. Haagerup, Operator valued weights in von Neumann algebras, J. Funct. Anal., II, 32 (1979), 175-206; 33 (1979), 339-361.

[3] __ L L L -spaces associated with an arbitrary von Neumann algebra.

[4] H. Halpern, Essential central spectrum and range for elements of a von Neumann algebra, Pacific J. Math., 43 (1972), 349-380.

[5] H. Halpern and V. Kaftal, Compact operators in type $\mathrm{III}_{\lambda}$ and type $\mathrm{III}_{0}$ factors, Math. Ann., 273 (1986), 251-270.

[6] _. Compact operators in type III $_{\lambda}$ and type III $_{0}$ factors, II, Tohôku Math. J., 39 (1987), 153-173.

[7] _ Finite weight projections in factor von Neumann algebras, Proc. Sympos. Pure Math., vol. 51, Amer. Math. Soc., Providence, R.I., 1990.

[8] R. V. Kadison and J. R. Ringrose, Fundamentals of the Theory of Operator Algebras Volumes I and II, Academic Press, Orlando, 1986.

[9] I. Kaplansky, Projections in Banach algebras, Ann. Math., 53 (1951), 235-249.

[10] G. Pedersen, $C^{*}$-Algebras and their Automorphism Groups. Academic Press, London, 1979.

[11] G. Pedersen and M. Takesaki, The Radon Nikodym theorem for von Neumann algebras, Acta Math., 130 (1973), 53-88.

[12] F. Riesz and B. Sz.-Nagy, Functional Analysis, F. Ungar Publ., New York, 1965.

[13] S. Stratila, Modular Theory of von Neumann Algebras, Abacus Press, Bucharest, 1981.

[14] S. Stratila and L. Zsidó, An algebraic reduction theory of $W^{*}$-algebras, I, II, J. Funct. Anal., 11 (1972), 295-313, and Rev. Roum. Math. Pures Appl., 18 (1973), 407-460.

[15] S. Stratila and L. Zsidó, Lectures in von Neumann Algebras, Turnbridge Abacus Press, Wells, 1981.

[16] W. I. M. Wils, Two sided ideals in $W^{*}$-algebras, J. Reine Angew. Math., 244 (1970), 55-68.

Received February 24, 1989.

UNIVERSITY OF CINCINNATI

CinCINNATI, OH 45221

AND

UNIVERSITY OF STUTTGART

STUTTGART (80)

FEDERAL RePUblic of GeRMANY 



\section{PACIFIC JOURNAL OF MATHEMATICS EDITORS}

V. S. VARADARAJAN

(Managing Editor)

University of California

Los Angeles, CA 90024-1555-05

Herbert Clemens

University of Utah

Salt Lake City, UT 84112

THOMAS ENRIGHT

University of California, San Diego

La Jolla, CA 92093

\section{R. FINN}

Stanford University

Stanford, CA 94305

HermanN FlaschKa

University of Arizona

Tucson, AZ 85721

VAUGHAN F. R. JoNES

University of California

Berkeley, CA 94720

STEVEn KeRCKHOFF

Stanford University

Stanford, CA 94305
C. C. MOORE

University of California

Berkeley, CA 94720

MARTIN SCHARLEMANN

University of California

Santa Barbara, CA 93106

Harold STARK

University of California, San Diego La Jolla, CA 92093

\section{ASSOCIATE EDITORS}

R. ARENS

E. F. BECKENBACH (1906-1982)
B. H. NeumanN

\section{SUPI}

UNIVERSITY OF ARIZONA

UNIVERSITY OF BRITISH COLUMBIA

CALIFORNIA INSTITUTE OF TECHNOLOGY

UNIVERSITY OF CALIFORNIA

MONTANA STATE UNIVERSITY

UNIVERSITY OF NEVADA, RENO

NEW MEXICO STATE UNIVERSITY

OREGON STATE UNIVERSITY
F. WolF
K. YoshidA
(1904-1989)

UNIVERSITY OF OREGON UNIVERSITY OF SOUTHERN CALIFORNIA

STANFORD UNIVERSITY

UNIVERSITY OF HAWAII

UNIVERSITY OF TOKYO

UNIVERSITY OF UTAH

WASHINGTON STATE UNIVERSITY

UNIVERSITY OF WASHINGTON 


\section{Pacific Journal of Mathematics}

Vol. 147, No. $1 \quad$ January, 1991

Mark S. Ashbaugh, Evans Malott Harrell, II and Roman Svirsky, On minimal and maximal eigenvalue gaps and their causes $\ldots \ldots \ldots \ldots \ldots 1$

Robert Coleman and Francis Oisin McGuinness, Rational formal group

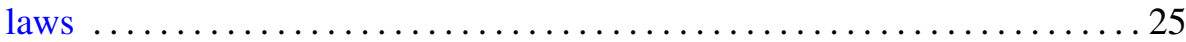

Jacek M. Cygan and Leonard Frederick Richardson, $D$-harmonic distributions and global hypoellipticity on nilmanifolds ...........29

Satya Deo and Kalathoor Varadarajan, Some examples of nontaut

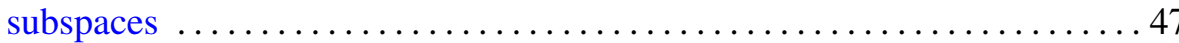

Maria Fragoulopoulou, Automatic continuity of *-morphisms between

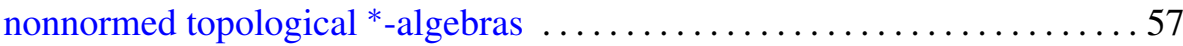

Stephen J. Gardiner, Removable singularities for subharmonic functions . . . 71 Herbert Paul Halpern, Victor Kaftal and László Zsidó, Finite weight projections in von Neumann algebras $\ldots \ldots \ldots \ldots \ldots \ldots \ldots \ldots \ldots \ldots$

Telemachos E. Hatziafratis, Explicit $\bar{\partial}$-primitives of Henkin-Leiterer kernels on Stein manifolds

Ka Hin Leung, A construction of an ordered division ring with a rank one

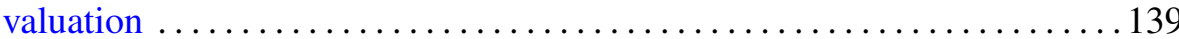

Christopher K. McCord, Nielsen numbers and Lefschetz numbers on solvmanifolds

Katsuro Sakai and Raymond Y. T. Wong, Manifold subgroups of the homeomorphism group of a compact $Q$-manifold

Caroline Perkins Sweezy, $L$-harmonic functions and the exponential square class 